\title{
Lepton-flavored scalar dark matter with minimal flavor violation
}

\author{
Chao-Jung Lee and Jusak Tandean \\ Department of Physics and Center for Theoretical Sciences, National Taiwan University, \\ Taipei 106, Taiwan \\ E-mail: r02222074@ntu.edu.tw, jtandean@yahoo.com
}

ABSTRACT: We explore scalar dark matter that is part of a lepton flavor triplet satisfying symmetry requirements under the hypothesis of minimal flavor violation. Beyond the standard model, the theory contains in addition three right-handed neutrinos that participate in the seesaw mechanism for light neutrino mass generation. The dark-matter candidate couples to standard-model particles via Higgs-portal renormalizable interactions as well as to leptons through dimension-six operators, all of which have minimal flavor violation built-in. We consider restrictions on the new scalars from the Higgs boson measurements, observed relic density, dark-matter direct detection experiments, LEP II measurements on $e^{+} e^{-}$scattering into a photon plus missing energy, and searches for flavor-violating lepton decays. The viable parameter space can be tested further with future data. Also, we investigate the possibility of the new scalars' couplings accounting for the tentative hint of Higgs flavor-violating decay $h \rightarrow \mu \tau$ recently detected in the CMS experiment. They are allowed by constraints from other Higgs data to produce a rate of this decay roughly compatible with the CMS finding.

Keywords: Cosmology of Theories beyond the SM, Discrete and Finite Symmetries, Neutrino Physics, Higgs Physics

ARXIV EPRINT: 1410.6803 


\section{Contents}

1 Introduction 1

2 Minimal lepton flavor violation framework $\quad 2$

3 Lepton-flavored dark matter 5

4 Numerical analysis $\quad 7$

$\begin{array}{lll}4.1 & \text { Higgs-portal interactions } & 7\end{array}$

$\begin{array}{llr}4.2 & \text { Effective DM-lepton interactions } & 9\end{array}$

$\begin{array}{lll}4.3 & \text { Flavor-violating Higgs decay } & 14\end{array}$

5 Conclusions 16

$\begin{array}{ll}\text { A Cross section of } e^{+} e^{-} \rightarrow \gamma S_{k} S_{l}^{*} & 17\end{array}$

$\begin{array}{ll}\text { B Decays of } S \text { particles } & 18\end{array}$

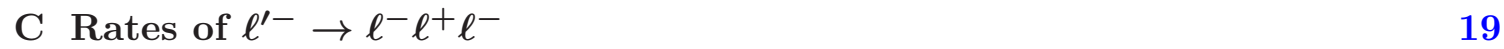

\section{Introduction}

It is now widely accepted that dark matter (DM) exists in the Universe. Many observations have led to the inference that DM makes up almost $27 \%$ of the cosmic energy density budget [1]. In spite of the evidence, however, the identity of the basic constituents of DM has continued to be elusive, with the data suggesting that new physics beyond the standard model (SM) is needed to account for it [2-4].

The necessity for invoking new physics is even more obvious in the treatment of neutrinos. Since they stay massless in the SM, it cannot explain the numerous measurements of nonzero neutrino mass and mixing [1]. Another longstanding and related conundrum is whether neutrinos are Dirac or Majorana particles.

In the absence of clear empirical guidance about how to address these problems, it is of interest to entertain various possibilities. Among the most appealing are models that link the DM and neutrino sectors in such a way that solves the puzzles in an interconnected or unified manner. In this paper, we explore a scenario along a similar line, where DM carries lepton-flavor quantum numbers and its interactions have some linkage to what makes neutrinos massive. To make the neutrino connection, we adopt the framework of so-called minimal flavor violation (MFV).

Motivated by the fact that the SM has been very successful in describing the existing data on flavor-changing neutral currents and $C P$-violating processes in the quark sector, 
the MFV hypothesis postulates that Yukawa couplings are the only sources for the breaking of flavor and $C P$ symmetries [5-13]. Its application to the study of DM carrying quarkflavor quantum numbers was first proposed in ref. [14]. The stability of the quark-flavored $\mathrm{DM}$ is due to the presence of an accidental discrete symmetry which is an element of the combined color and quark-flavor group under the MFV assumption [15].

Although the implementation of MFV for quarks is straightforward, there is no unique way to extend the notion of MFV to the lepton sector, as the SM by itself does not accommodate lepton-flavor violation. Since significant flavor mixing among neutrinos has been measured, it is interesting to formulate MFV for leptons by incorporating ingredients beyond the SM that can account for this observation [13]. Thus, here we consider the SM slightly expanded with the addition of three right-handed neutrinos plus a lepton-flavor triplet of scalar fields which has transformation properties satisfying the MFV principle and contains DM of the popular weakly interacting massive particle (WIMP) type. The right-handed neutrinos allow us to activate the usual type-I seesaw mechanism which results in Majorana neutrinos with small masses [16-24]. We will not focus on the less interesting possibility of Dirac neutrinos. Another difference from the quark case is that MFV does not in general lead to longevity for lepton-flavored DM because of lack of a counterpart of the accidental symmetry which keeps quark-flavored DM stable [15]. Therefore, to ensure the stability of our DM candidate we impose a $Z_{2}$ symmetry under which the triplet scalars are odd and other particles even.

In the next section, we briefly review the MFV framework in the lepton sector. In section 3, we describe the Lagrangians with MFV built-in for the scalar triplet. We assign its quantum numbers in analogy to its quark-flavor counterpart discussed in the literature [14, 25]. Accordingly, the triplet can interact with SM particles via a Higgsboson portal at the renormalizable level and also couple to SM leptons through effective dimension-six operators. Section 4 contains our numerical analysis. We explore constraints on the two types of DM-SM interactions from the Higgs boson data, observed relic abundance, DM direct detection experiments, LEP II measurements of $e^{+} e^{-}$collisions into a photon plus missing energy, and searches for flavor-violating charged lepton decays. In addition, we examine whether the new scalars' interactions can explain the recent potential indication from the CMS experiment of the Higgs flavor-violating decay $h \rightarrow \mu \tau$ which would be an unmistakable signal of physics beyond the SM if confirmed by future measurements. We make our conclusions in section 5. Some lengthy formulas are relegated to a few appendices.

\section{Minimal lepton flavor violation framework}

In the SM supplemented with three right-handed neutrinos, the renormalizable Lagrangian for lepton masses can be written as

$$
\mathcal{L}_{\mathrm{m}}=-\left(Y_{\nu}\right)_{k l} \bar{L}_{k, L} \nu_{l, R} \tilde{H}-\left(Y_{e}\right)_{k l} \bar{L}_{k, L} E_{l, R} H-\frac{1}{2}\left(M_{\nu}\right)_{k l} \overline{\nu_{k, R}^{\mathrm{c}}} \nu_{l, R}+\text { H.c. },
$$

where $k, l=1,2,3$ are summed over, $L_{k, L}$ represents left-handed lepton doublets, $\nu_{l, R}$ $\left(E_{l, R}\right)$ denotes right-handed neutrinos (charged leptons), $Y_{\nu, e}$ are matrices for the Yukawa 
couplings, $H$ is the Higgs doublet, $\tilde{H}=i \tau_{2} H^{*}$, and $M_{\nu}$ is the Majorana mass matrix for $\nu_{l, R}$. The $M_{\nu}$ part is essential for the type-I seesaw mechanism to generate light neutrino masses [16-24].

If neutrinos are Dirac fermions, the $M_{\nu}$ terms are absent from eq. (2.1), and the MFV hypothesis [13] then implies that $\mathcal{L}_{\mathrm{m}}$ has formal invariance under the global group $\mathrm{U}(3)_{L} \times \mathrm{U}(3)_{\nu} \times \mathrm{U}(3)_{E}=G_{\ell} \times \mathrm{U}(1)_{L} \times \mathrm{U}(1)_{\nu} \times \mathrm{U}(1)_{E}$, with $G_{\ell}=\mathrm{SU}(3)_{L} \times \mathrm{SU}(3)_{\nu} \times \mathrm{SU}(3)_{E}$ being the flavor symmetry. This entails that $L_{k, L}, \nu_{k, R}$, and $E_{k, R}$ transform as fundamental representations of $\mathrm{SU}(3)_{L, \nu, E}$, respectively,

$$
L_{L} \rightarrow V_{L} L_{L}, \quad \nu_{R} \rightarrow V_{\nu} \nu_{R}, \quad E_{R} \rightarrow V_{E} E_{R}, \quad V_{L, \nu, E} \in \mathrm{SU}(3)_{L, \nu, E},
$$

whereas the Yukawa couplings transform in the spurion sense according to

$$
Y_{\nu} \rightarrow V_{L} Y_{\nu} V_{\nu}^{\dagger}, \quad Y_{e} \rightarrow V_{L} Y_{e} V_{E}^{\dagger} .
$$

Taking advantage of the symmetry under $G_{\ell}$, we work in the basis where

$$
Y_{e}=\frac{\sqrt{2}}{v} \operatorname{diag}\left(m_{e}, m_{\mu}, m_{\tau}\right),
$$

with $v \simeq 246 \mathrm{GeV}$ being the vacuum expectation value of $H$, and the fields $\nu_{k, L}, \nu_{k, R}, E_{k, L}$, and $E_{k, R}$ refer to the mass eigenstates. We can then express $L_{k, L}$ and $Y_{\nu}$ in terms of the Pontecorvo-Maki-Nakagawa-Sakata (PMNS $[26,27])$ neutrino mixing matrix $U_{\text {PMNS }}$ as

$$
L_{k, L}=\left(\begin{array}{c}
\left(U_{\mathrm{PMNS}}\right)_{k l} \nu_{l, L} \\
E_{k, L}
\end{array}\right), \quad Y_{\nu}=\frac{\sqrt{2}}{v} U_{\mathrm{PMNS}} \hat{m}_{\nu}, \quad \hat{m}_{\nu}=\operatorname{diag}\left(m_{1}, m_{2}, m_{3}\right),
$$

where $m_{1,2,3}$ are the light neutrino eigenmasses and in the standard parametrization [1]

$$
U_{\mathrm{PMNS}}=\left(\begin{array}{ccc}
c_{12} c_{13} & s_{12} c_{13} & s_{13} e^{-i \delta} \\
-s_{12} c_{23}-c_{12} s_{23} s_{13} e^{i \delta} & c_{12} c_{23}-s_{12} s_{23} s_{13} e^{i \delta} & s_{23} c_{13} \\
s_{12} s_{23}-c_{12} c_{23} s_{13} e^{i \delta} & -c_{12} s_{23}-s_{12} c_{23} s_{13} e^{i \delta} & c_{23} c_{13}
\end{array}\right)
$$

with $\delta$ being the $C P$ violation phase, $c_{k l}=\cos \theta_{k l}$, and $s_{k l}=\sin \theta_{k l}$.

If neutrinos are Majorana in nature, $Y_{\nu}$ must be modified. The presence of $M_{\nu}$ in eq. (2.1) with nonzero elements much bigger than those of $v Y_{\nu} / \sqrt{2}$ activates the seesaw mechanism [16-24], leading to the light neutrinos' mass matrix

$$
m_{\nu}=-\frac{v^{2}}{2} Y_{\nu} M_{\nu}^{-1} Y_{\nu}^{\mathrm{T}}=U_{\mathrm{PMNS}} \hat{m}_{\nu} U_{\mathrm{PMNS}}^{\mathrm{T}}
$$

where now $U_{\mathrm{PMNS}}$ contains the diagonal matrix $P=\operatorname{diag}\left(e^{i \alpha_{1} / 2}, e^{i \alpha_{2} / 2}, 1\right)$ multiplied from the right and involving the Majorana phases $\alpha_{1,2}$. This allows one to write [28]

$$
Y_{\nu}=\frac{i \sqrt{2}}{v} U_{\mathrm{PMNS}} \hat{m}_{\nu}^{1 / 2} O M_{\nu}^{1 / 2}
$$


where $O$ is in general a complex matrix satisfying $O O^{\mathrm{T}}=\mathbb{1}$, the right-hand side being a $3 \times 3$ unit matrix, and $M_{\nu}=\operatorname{diag}\left(M_{1}, M_{2}, M_{3}\right)$. From this point on, we assume that neutrinos are Majorana particles and entertain the possibility that the right-handed neutrinos are degenerate, so that $M_{\nu}=\mathcal{M} \mathbb{1}$ with $\mathcal{M}$ being their mass. In this scenario, the $M_{\nu}$ part of $\mathcal{L}_{\mathrm{m}}$ breaks $\mathrm{SU}(3)_{\nu}$ into $\mathrm{O}(3)_{\nu}$, and as a consequence we have $G_{\ell}=\mathcal{G}_{\ell} \times \mathrm{O}(3)_{\nu}$, where $\mathcal{G}_{\ell}=\mathrm{SU}(3)_{L} \times \mathrm{SU}(3)_{E}$ is the pertinent flavor group after the heavy right-handed neutrinos are integrated out [13].

To put together Lagrangians beyond the SM with MFV built-in, one inserts $Y_{\nu, e}, Y_{\nu, e}^{\dagger}$, and their products among SM and new fields to construct $\mathcal{G}_{\ell}$-invariant operators that are singlet under the SM gauge group $[12,13]$. Of interest here are the matrix products $\mathrm{A}=Y_{\nu} Y_{\nu}^{\dagger}$ and $\mathrm{B}=Y_{e} Y_{e}^{\dagger}$, which transform as $(1 \oplus 8,1)$ under $\mathcal{G}_{\ell}$, as $Y_{\nu}$ and $Y_{e}$ transform as $(3,1)$ and $(3, \overline{3})$, respectively. In a model-independent approach, combinations of $\mathrm{A}$ and $\mathrm{B}$ are collected into an object $\Delta$ which formally comprises an infinite number of terms, namely $\Delta=\sum \xi_{j k l \ldots} \mathrm{A}^{j} \mathrm{~B}^{k} \mathrm{~A}^{l} \ldots$ with coefficients $\xi_{j k l \ldots}$ expected to be at most of $\mathcal{O}(1)$. Under the MFV hypothesis, $\xi_{j k l \ldots}$ are real because complex $\xi_{j k l . .}$ would introduce new $C P$-violation sources beyond that in the Yukawa couplings. With the Cayley-Hamilton identity $X^{3}=X^{2} \operatorname{Tr} X+\frac{1}{2} X\left[\operatorname{Tr} X^{2}-(\operatorname{Tr} X)^{2}\right]+\mathbb{1} \operatorname{Det} X$ for an invertible $3 \times 3$ matrix $X$, one can resum the infinite series into a finite number of terms [29, 30]:

$$
\begin{aligned}
\Delta= & \xi_{1} \mathbb{1}+\xi_{2} \mathrm{~A}+\xi_{3} \mathrm{~B}+\xi_{4} \mathrm{~A}^{2}+\xi_{5} \mathrm{~B}^{2}+\xi_{6} \mathrm{AB}+\xi_{7} \mathrm{BA}+\xi_{8} \mathrm{ABA}+\xi_{9} \mathrm{BA}^{2}+\xi_{10} \mathrm{BAB} \\
& +\xi_{11} \mathrm{AB}^{2}+\xi_{12} \mathrm{ABA}^{2}+\xi_{13} \mathrm{~A}^{2} \mathrm{~B}^{2}+\xi_{14} \mathrm{~B}^{2} \mathrm{~A}^{2}+\xi_{15} \mathrm{~B}^{2} \mathrm{AB}+\xi_{16} \mathrm{AB}^{2} \mathrm{~A}^{2}+\xi_{17} \mathrm{~B}^{2} \mathrm{~A}^{2} \mathrm{~B} .
\end{aligned}
$$

Although $\xi_{i j k \ldots}$ are real, the reduction of the infinite series into the 17 terms can make the coefficients $\xi_{r}$ in eq. (2.9) complex due to imaginary parts among the traces of the matrix products $A^{i} B^{j} A^{k} \cdots$. Such imaginary contributions turn out to be small [29-32], and so hereafter we ignore $\operatorname{Im} \xi_{r}$.

In the Dirac neutrino case, $Y_{\nu}$ in eq. (2.5) leads to $\mathrm{A}=2 U_{\mathrm{PMNS}} \hat{m}_{\nu}^{2} U_{\mathrm{PMNS}}^{\dagger} / v^{2}$, which has tiny elements. In contrast, if neutrinos are of Majorana nature,

$$
\mathrm{A}=\frac{2}{v^{2}} U_{\mathrm{PMNS}} \hat{m}_{\nu}^{1 / 2} O M_{\nu} O^{\dagger} \hat{m}_{\nu}^{1 / 2} U_{\mathrm{PMNS}}^{\dagger}
$$

from eq. (2.8), and so A can have much greater elements if the right-handed neutrinos' mass $\mathcal{M}$ in $M_{\nu}$ is sufficiently large. Since as an infinite series $\Delta$ has to converge, $\mathcal{M}$ cannot be arbitrarily large [29-32]. Accordingly, we require the largest eigenvalue of $\mathrm{A}$ to be unity, which implies that the elements of $\mathrm{B}=Y_{e} Y_{e}^{\dagger}$ are small compared to those of $\mathrm{A}$ and that, consequently, we can drop most of the terms in eq. (2.9) except the first few. It follows that in this study

$$
\Delta=\xi_{1} \mathbb{1}+\xi_{2} \mathrm{~A}+\xi_{4} \mathrm{~A}^{2}=\Delta^{\dagger}
$$




\section{Lepton-flavored dark matter}

The new sector of the theory also includes three complex scalar fields which are singlet under the SM gauge group and constitute a triplet under $\mathcal{G}_{\ell}=\mathrm{SU}(3)_{L} \times \mathrm{SU}(3)_{E}$, namely ${ }^{1}$

$$
\tilde{s}=\left(\begin{array}{l}
\tilde{s}_{1} \\
\tilde{s}_{2} \\
\tilde{s}_{3}
\end{array}\right) \sim(3,1) .
$$

To maintain the longevity of its lowest-mass eigenstate as the DM candidate, we invoke a $Z_{2}$ symmetry under which $\tilde{s}$ is odd and other particles are even. ${ }^{2}$ This will disallow $\mathcal{G}_{\ell}$-invariant interaction terms involving odd numbers of $\tilde{s}_{k}^{(*)}$ that could cause the DM state to decay.

It follows that the renormalizable Lagrangian for the interactions of the scalar fields with one another and the SM gauge bosons is given by

$$
\begin{aligned}
\mathcal{L}= & \left(\mathcal{D}^{\eta} H\right)^{\dagger} \mathcal{D}_{\eta} H+\partial^{\eta} \tilde{s}^{\dagger} \partial_{\eta} \tilde{s}-\mathcal{V}, \\
\mathcal{V}= & \mu_{H}^{2} H^{\dagger} H+\tilde{s}^{\dagger} \mu_{s}^{2} \tilde{s}+\lambda_{H}\left(H^{\dagger} H\right)^{2}+2 H^{\dagger} H \tilde{s}^{\dagger} \Delta_{H S} \tilde{s}+\left(\tilde{s}^{\dagger} \Delta_{S S} \tilde{s}\right)^{2} \\
\supset & \tilde{s}^{\dagger}\left(\mu_{s 0}^{2} \mathbb{1}+\mu_{s 1}^{2} \mathrm{~A}+\mu_{s 2}^{2} \mathrm{~A}^{2}\right) \tilde{s}+2 H^{\dagger} H \tilde{s}^{\dagger}\left(\lambda_{s 0} \mathbb{1}+\lambda_{s 1} \mathrm{~A}+\lambda_{s 2} \mathrm{~A}^{2}\right) \tilde{s} \\
& +\left[\tilde{s}^{\dagger}\left(\lambda_{s 0}^{\prime} \mathbb{1}+\lambda_{s 1}^{\prime} \mathrm{A}+\lambda_{s 2}^{\prime} \mathrm{A}^{2}\right) \tilde{s}\right]^{2},
\end{aligned}
$$

where $\mathcal{D}_{\eta}$ is the covariant derivative involving the gauge fields, $\mu_{s}^{2}$ and $\Delta_{H S, S S}$ are $3 \times 3$ matrices, and the Higgs doublet after electroweak symmetry breaking

$$
H=\left(\begin{array}{c}
0 \\
\frac{1}{\sqrt{2}}(h+v)
\end{array}\right),
$$

with $h$ being the physical Higgs field. The expression for $\mu_{s}^{2}\left(\Delta_{H S, S S}\right)$ has the form in eq. (2.11) up to an overall factor with mass dimension $2(0)$, and hence the parameters $\mu_{s j}^{2}$, $\lambda_{s j}$, and $\lambda_{s j}^{\prime}$ are real.

With $A$ being Hermitian, we have the relation $A=\mathcal{U} \operatorname{diag}\left(\hat{\mathrm{A}}_{1}, \hat{\mathrm{A}}_{2}, \hat{\mathrm{A}}_{3}\right) \mathcal{U}^{\dagger}$ where $\mathcal{U}$ is a unitary matrix and $\hat{A}_{k}$ denotes the eigenvalues of $A$. Accordingly, the matrices sandwiched between $\tilde{s}^{\dagger}$ and $\tilde{s}$ in eq. (3.3) can be simultaneously diagonalized. It follows that $\tilde{s}_{k}$ are related to the mass eigenstates $S_{k}$ by

$$
S=\left(\begin{array}{c}
S_{1} \\
S_{2} \\
S_{3}
\end{array}\right)=\mathcal{U}^{\dagger} \tilde{s},
$$

in terms of which

$$
\mathcal{L} \supset-m_{S_{k}}^{2} S_{k}^{*} S_{k}-\lambda_{k}\left(h^{2}+2 h v\right) S_{k}^{*} S_{k}-\left(\lambda_{k}^{\prime} S_{k}^{*} S_{k}\right)^{2},
$$

\footnotetext{
${ }^{1}$ Lepton flavor triplets with DM components have also been considered in the contexts of other models [33-35].

${ }^{2}$ Outside the MFV framework, it is possible to have a DM-stabilizing $Z_{2}$ symmetry that is a remnant of a lepton flavor group [36-39].
} 
where summation over $k$ is implicit,

$$
m_{S_{k}}^{2}=\mu_{k}^{2}+\lambda_{k} v^{2}, \quad \mu_{k}^{2}=\mu_{s 0}^{2}+\mu_{s 1}^{2} \hat{\mathrm{A}}_{k}+\mu_{s 2}^{2} \hat{\mathrm{A}}_{k}^{2}, \quad \lambda_{k}^{(\prime)}=\lambda_{s 0}^{(\prime)}+\lambda_{s 1}^{(\prime)} \hat{\mathrm{A}}_{k}+\lambda_{s 2}^{(\prime)} \hat{\mathrm{A}}_{k}^{2} .
$$

Since $\mu_{s i}^{2}$ and $\lambda_{s i}^{(\prime)}$ are free parameters, so are $m_{S_{k}}>0$ and $\lambda_{k}^{(\prime)}$. There are, however, theoretical restrictions on $\lambda_{k}^{(\prime)}$ as well as $\lambda_{H}$. The stability of the vacuum requires $\mathcal{V}$ to be bounded from below, which entails $\lambda_{H}>0,\left(\lambda_{k}^{\prime}\right)^{2}>0$, and $\lambda_{k}>-\sqrt{\lambda_{H}}\left|\lambda_{k}^{\prime}\right|$, the second inequality being automatically satisfied by the reality of $\lambda_{k}^{\prime}$. The condition of perturbativity $[40,41]$ translates into $\left|\lambda_{H, k}\right|<4 \pi$ and $\left(\lambda_{k}^{\prime}\right)^{2}<4 \pi$.

The $\lambda_{k}$ part in eq. (3.6) is responsible for the Higgs-portal interactions of the new scalars with SM particles. As we detail later, in this paper we select $S_{3}$ to be less massive than $S_{1,2}$ and serve as the DM candidate. In addition, we pick the $S_{1,2}$ masses to be sufficiently bigger than $m_{S_{3}}$ in order that their impact on the relic density can be ignored. In that case, $\lambda_{3}$ controls the Higgs-mediated annihilations of the DM into SM particles, its scattering off a nucleon via Higgs exchange, and also the Higgs nonstandard invisible decay if the $S_{3}$ mass is low enough. All of these processes are subject to constraints from various recent data.

Because of their flavor quantum numbers in eq. (3.1), the new particles cannot have renormalizable contact interactions with SM fermions. Rather, under the MFV framework supplemented with the DM stabilizing $Z_{2}$ symmetry, $S_{k}$ can couple with SM leptons due to effective operators of dimension six given by ${ }^{3}$

$$
\mathcal{L}^{\prime}=\frac{C_{b d k l}^{L}}{\Lambda^{2}} O_{b d k l}^{L}+\frac{C_{b d k l}^{R}}{\Lambda^{2}} O_{b d k l}^{R}+\left(\frac{C_{b d k l}^{L R}}{\Lambda^{2}} O_{b d k l}^{L R}+\text { H.c. }\right),
$$

where summation over $b, d, k, l=1,2,3$ is implicit,

$$
\begin{array}{ll}
C_{b d k l}^{L}=\left(\Delta_{L L}\right)_{b d}\left(\Delta_{S S}\right)_{k l}+\left(\Delta_{L S}\right)_{b l}\left(\Delta_{S L}\right)_{k d}+\left(\Delta_{L S}\right)_{k d}\left(\Delta_{S L}\right)_{b l}, & O_{b d k l}^{L}=i \bar{L}_{b, L} \gamma^{\rho} L_{d, L} \tilde{s}_{k}^{*} \stackrel{\leftrightarrow}{\partial}_{\rho} \tilde{s}_{l}, \\
C_{b d k l}^{R}=\delta_{b d}\left(\Delta_{S S}^{\prime}\right)_{k l}, & O_{b d k l}^{R}=i \bar{E}_{b, R} \gamma^{\rho} E_{d, R} \tilde{s}_{k}^{*} \stackrel{\leftrightarrow}{\partial} \tilde{s}_{l}, \\
C_{b d k l}^{L R}=\left(\Delta_{L Y} Y_{e}\right)_{b d}\left(\Delta_{S S}^{\prime \prime}\right)_{k l}+\left(\Delta_{L S}^{\prime}\right)_{b l}\left(\Delta_{S Y} Y_{e}\right)_{k d}, & O_{b d k l}^{L R}=\bar{L}_{b, L} E_{d, R} \tilde{s}_{k}^{*} \tilde{s}_{l} H,
\end{array}
$$

with $^{4} X \overleftrightarrow{\leftrightarrow}_{\rho} Y=X \partial_{\rho} Y-\partial_{\rho} X Y$ and $\tilde{s}_{k}=\mathcal{U}_{k l} S_{l}$. We have dropped terms in $C^{R}$ that are suppressed by two powers of $Y_{e}$. Since the right-handed neutrinos have masses far exceeding the $\mathrm{TeV}$ level, we do not include operators involving them in $\mathcal{L}^{\prime}$. The mass scale $\Lambda$ characterizes the heavy new physics underlying these interactions and also responsible for the Lorentz and flavor structure of the operators. Specifically, $O^{L, R}\left(O^{L R}\right)$ could arise from the exchange of a spin-one boson (scalar or fermion), and so $\Lambda$ would depend on its couplings and mass.

\footnotetext{
${ }^{3}$ Without the $Z_{2}$ symmetry, the DM candidate could undergo rapid decay triggered by effective operators involving odd numbers of $\tilde{s}$, such as $\epsilon_{b d k} \overline{\left(\Delta_{1} L_{L}\right)_{b}^{\mathrm{c}}} \tilde{H}^{*} \tilde{H}^{\dagger}\left(\Delta_{2} L_{L}\right)_{d}\left(\Delta_{3} \tilde{s}\right)_{k}$, where $\Delta_{1,2,3}$ are of the form in eq. (2.11) with their respective coefficients $\xi$ 's.

${ }^{4}$ The counterparts of $O^{L, E}$ with $\tilde{s}_{k}^{*} \overleftrightarrow{\partial}_{\rho} \tilde{s}_{l}$ replaced by $\tilde{s}_{k}^{*} \partial_{\rho} \tilde{s}_{l}+\partial_{\rho} \tilde{s}_{k}^{*} \tilde{s}_{l}$ are not independent and can be expressed in terms of $O^{L R(\dagger)}$ after partial integration and use of the lepton equations of motion [42].
} 
The $\Delta$ 's in $C^{L, R, L R}$ above are of the same form as in eq. (2.11), but have generally different coefficients $\xi$ 's. These $\xi$ 's are expected to be at most of $\mathcal{O}(1)$, and some of them may be suppressed or vanish, depending on the underlying theory. In our modelindependent approach with MFV, we single out a few of them in order to illustrate some of the phenomenological implications.

\section{Numerical analysis}

With $S_{3}$ being the DM, the cross section $\sigma_{\text {ann }}$ of $S_{3} S_{3}^{*}$ annihilation into SM particles needs to yield the present-day DM density $\Omega$. The two quantities are approximately related by [43]

$$
\Omega \hat{h}^{2}=\frac{2.14 \times 10^{9} x_{f} \mathrm{GeV}^{-1}}{\sqrt{g_{*}} m_{\mathrm{Pl}}\left(\hat{a}+3 \hat{b} / x_{f}\right)}, \quad x_{f}=\ln \frac{0.038 m_{S_{3}} m_{\mathrm{Pl}}\left(\hat{a}+6 \hat{b} / x_{f}\right)}{\sqrt{g_{*} x_{f}}},
$$

where $\hat{h}$ stands for the Hubble parameter, $m_{\mathrm{Pl}}=1.22 \times 10^{19} \mathrm{GeV}$ is the Planck mass, $g_{*}$ is the number of relativistic degrees of freedom below the freeze-out temperature $T_{f}=$ $m_{S_{3}} / x_{f}$, and $\hat{a}$ and $\hat{b}$ are defined by the expansion of the annihilation rate $\sigma_{\text {ann }} v_{\text {rel }}=\hat{a}+\hat{b} v_{\text {rel }}^{2}$ in terms of the relative speed $v_{\text {rel }}$ of the nonrelativistic $S_{3} S_{3}^{*}$ pair in their center-of-mass (c.m.) frame. The $\Omega$ expression takes into account the fact that the DM is a complex scalar particle.

\subsection{Higgs-portal interactions}

The $S_{3}$ contributions to $\sigma_{\text {ann }}$ originate mainly from the $\lambda_{3}$ term in eq. (3.6) as well as from the dimension- 6 operators in eq. (3.8). We consider first the possibility that the latter are absent. The $\lambda_{3}$ coupling gives rise to Higgs-mediated $S_{3} S_{3}^{*}$ collisions into SM particles, just as in the case of the SM-singlet scalar DM [44-50]. The resulting annihilation rate in the nonrelativistic limit is dominated by its $\hat{a}$ part,

$$
\sigma_{\mathrm{ann}} v_{\mathrm{rel}} \simeq \hat{a}=\frac{4 \lambda_{3}^{2} v^{2} m_{S_{3}}^{-1} \sum_{i} \Gamma\left(\tilde{h} \rightarrow X_{i}\right)}{\left(4 m_{S_{3}}^{2}-m_{h}^{2}\right)^{2}+\Gamma_{h}^{2} m_{h}^{2}}
$$

where $m_{h}$ is the mass of the Higgs boson, $\Gamma_{h}$ is its total width $\Gamma_{h}^{\mathrm{SM}}$ in the SM plus the rates of the decays $h \rightarrow S_{k} S_{k}^{*}$ to be discussed below, $\tilde{h}$ is a virtual Higgs boson having the same couplings as the physical $h$, but with the invariant mass $\sqrt{s}=2 m_{S_{3}}$, and $\tilde{h} \rightarrow X_{i}$ is any kinematically allowed decay mode of $\tilde{h}$. For $m_{S_{3}}>m_{h}$, the $S_{3} S_{3}^{*} \rightarrow h h$ reaction can happen, due to $s^{-}, t$-, and $u$-channel as well as contact diagrams, and hence needs to be included in $\hat{a}$. Numerically, we employ $m_{h}=125.1 \mathrm{GeV}$, which reflects the average of the most recent measurements [51, 52], and $\Gamma_{h}^{\mathrm{SM}}=4.08 \mathrm{MeV}$ [53]. Once the $\lambda_{3}$ values which reproduce the observed relic abundance are extracted, they need to fulfill important restrictions which we now address.

A number of underground experiments have been performed to detect WIMP DM directly by looking for the recoil energy of nuclei caused by the elastic scattering of a 


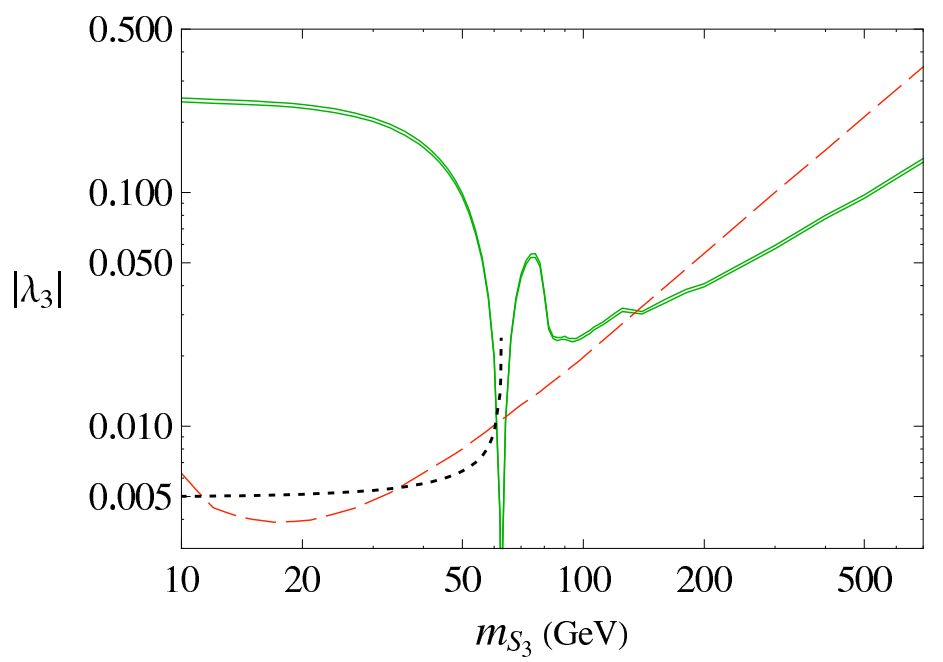

Figure 1. Values of $\left|\lambda_{3}\right|$ consistent with the relic density data (green solid curve), compared to upper limits on $\left|\lambda_{3}\right|$ from Higgs measurements (black dotted curve) and from null results of DM direct searches (red dashed curve), as discussed in the text.

WIMP off a nucleon, $N$. Our process of interest is $S_{3}^{(*)} N \rightarrow S_{3}^{(*)} N$ which proceeds mainly via Higgs exchange in the $t$ channel and hence depends on $\lambda_{3}$ as well. Its cross section is

$$
\sigma_{\mathrm{el}}=\frac{\lambda_{3}^{2} g_{N N h}^{2} m_{N}^{2} v^{2}}{\pi\left(m_{S_{3}}+m_{N}\right)^{2} m_{h}^{4}}
$$

in the nonrelativistic limit, where $m_{N}$ is the nucleon mass and $g_{N N h}$ denotes the Higgsnucleon effective coupling whose value is within the range $0.0011 \leq g_{N N h} \leq 0.0032[49,50]$. The null result of searches by the LUX experiment [54] translates into the strictest limit to date on $\sigma_{\mathrm{el}}$.

If $m_{S_{k}}$ is less than half of the Higgs mass, the nonstandard decay channel $h \rightarrow S_{k} S_{k}^{*}$ is open. This leads to the branching ratio

$$
\mathcal{B}\left(h \rightarrow S^{*} S\right)=\frac{\sum_{k} \Gamma_{h \rightarrow S_{k}^{*} S_{k}}}{\Gamma_{h}^{\mathrm{SM}}+\sum_{k} \Gamma_{h \rightarrow S_{k}^{*} S_{k}}} .
$$

where the summation is over final states satisfying $2 m_{S_{k}}<m_{h}$ and from eq. (3.6)

$$
\Gamma_{h \rightarrow S_{k}^{*} S_{k}}=\frac{\lambda_{k}^{2} v^{2}}{4 \pi m_{h}} \sqrt{1-\frac{4 m_{S_{k}}^{2}}{m_{h}^{2}}} .
$$

The couplings $\lambda_{k}$ are thus subject to restrictions on the Higgs invisible or non-SM decay modes from collider data.

To determine the $\lambda_{3}$ values that are consistent with the observed relic density, we apply the relevant formulas described above and impose $0.1155 \leq \Omega \hat{h}^{2} \leq 0.1241$ which is the $90 \%$ confidence level (CL) range of the data $\Omega \hat{h}^{2}=0.1198 \pm 0.0026$ [1]. In figure 1 we display the result (green solid curve) for $m_{S_{3}} \geq 10 \mathrm{GeV}$. It needs to be compared with 
the red dashed curve, which represents the upper limit on $\left|\lambda_{3}\right|$ inferred from the null result reported by the LUX collaboration [54]. To arrive at this curve, we used eq. (4.3) with $g_{N N h}=0.0011$, which is the lower end of its range and thus leads to the loosest limit on $\left|\lambda_{3}\right|$ from the most stringent of DM direct searches to date. For $2 m_{S_{3}}<m_{h}$, the experimental information on the Higgs nonstandard invisible decay implies further restraints. Assuming that the channels $h \rightarrow S_{1} S_{1}^{*}, S_{2} S_{2}^{*}$ are absent, we have plotted the black dotted curve upon demanding $\mathcal{B}\left(h \rightarrow S^{*} S\right)<0.19$ based on the bounds from the latest analyses of collider data [55-59]. The opening of the $S_{1,2} S_{1,2}^{*}$ channels would cause the dotted curve to shift down.

From the figure, one can infer that the $\lambda_{3}$ contribution to the annihilation rate is much less than half of the required amount if $m_{S_{3}}<90 \mathrm{GeV}$, except the neighborhood of $m_{S_{3}}=m_{h} / 2$. In other words, over most of this mass region the $\lambda_{3}$ term in eq. (3.6) cannot play the leading role responsible for the observed relic abundance. Therefore, the dominant contribution must come from the effective interactions in eq. (3.8), absent other DM candidates. For larger $m_{S_{3}}$, on the other hand, each of the two sources can generate a nonneglible effect on the relic density.

\subsection{Effective DM-lepton interactions}

The effective operators in eq. (3.8) induce DM annihilations into SM leptons and are subject to constraints which may not apply to the Higgs- $S_{k}$ renormalizable couplings. From eq. (3.9), we derive the amplitudes for the DM annihilation $S_{3}(p) S_{3}^{*}(\bar{p}) \rightarrow \ell_{b}^{-} \ell_{d}^{+}, \nu_{b} \nu_{d}$ to be

$$
\begin{aligned}
\mathcal{M}_{S_{3} \bar{S}_{3} \rightarrow \ell_{b} \bar{\ell}_{d}} & =\frac{1}{\Lambda^{2}} \bar{u}_{b}\left[\frac{-v}{\sqrt{2}}\left(\mathcal{C}_{b d}^{L R} P_{R}+\mathcal{C}_{d b}^{L R *} P_{L}\right)+\gamma^{\rho}(\bar{p}-p)_{\rho}\left(\mathcal{C}_{b d}^{L} P_{L}+\mathcal{C}_{b d}^{R} P_{R}\right)\right] v_{d}, \\
\mathcal{M}_{S_{3} \bar{S}_{3} \rightarrow \nu_{b} \nu_{d}} & =\frac{1}{\Lambda^{2}}(\bar{p}-p)_{\rho} \bar{u}_{b} \gamma^{\rho}\left(\mathcal{C}_{b d}^{L} P_{L}-\mathcal{C}_{d b}^{L} P_{R}\right) v_{d},
\end{aligned}
$$

where $u_{b}$ and $v_{d}$ are the leptons' spinors, $P_{L, R}=\frac{1}{2}\left(1 \mp \gamma_{5}\right)$,

$$
\mathcal{C}_{b d}^{\epsilon}=\sum_{k, l} \mathcal{U}_{k 3}^{*} \mathcal{U}_{l 3} C_{b d k l}^{\epsilon}, \quad \epsilon=L R, L, R,
$$

and for the $\nu_{b} \nu_{d}$ channel we have taken into account the neutrinos' Majorana nature. The contributions of these reactions to the annihilation rate $\sigma_{\mathrm{ann}} v_{\text {rel }}=\hat{a}+\hat{b} v_{\text {rel }}^{2}$ are

$$
\begin{aligned}
\hat{a}= & \frac{\mathcal{K}^{\frac{1}{2}}\left(4 m_{S_{3}}^{2}, m_{\ell_{o}}^{2}, m_{\ell_{r}}^{2}\right) v^{2}}{256 \Lambda^{4} \pi m_{S_{3}}^{4}}\left[\left(\left|\mathcal{C}_{o r}^{L R}\right|^{2}+\left|\mathcal{C}_{r o}^{L R}\right|^{2}\right)\left(4 m_{S_{3}}^{2}-m_{\ell_{o}}^{2}-m_{\ell_{r}}^{2}\right)-4 \operatorname{Re}\left(\mathcal{C}_{o r}^{L R} \mathcal{C}_{r o}^{L R}\right) m_{\ell_{o}} m_{\ell_{r}}\right], \\
\hat{b}= & \frac{\mathcal{K}^{\frac{1}{2}}\left(4 m_{S_{3}}^{2}, m_{\ell_{o}}^{2}, m_{\ell_{r}}^{2}\right)}{1536 \Lambda^{4} \pi m_{S_{3}}^{4}}\left\{\left(\left|\mathcal{C}_{o r}^{L}\right|^{2}+\left|\mathcal{C}_{o r}^{R}\right|^{2}\right)\left[32 m_{S_{3}}^{4}-4\left(m_{\ell_{o}}^{2}+m_{\ell_{r}}^{2}\right) m_{S_{3}}^{2}-\left(m_{\ell_{o}}^{2}-m_{\ell_{r}}^{2}\right)^{2}\right]\right. \\
& \left.+48 \operatorname{Re}\left(\mathcal{C}_{o r}^{L *} \mathcal{C}_{o r}^{R}\right) m_{S_{3}}^{2} m_{\ell_{o}} m_{\ell_{r}}\right\} \\
& +\frac{\left|\mathcal{C}_{o r}^{L}\right|^{2} m_{S_{3}}^{2}}{12 \Lambda^{4} \pi}+\left(\mathcal{C}_{o r, r o}^{L R} \text { terms }\right),
\end{aligned}
$$

where $\mathcal{K}(x, y, z)=x^{2}+y^{2}+z^{2}-2(x y+y z+x z)$ and summation over $o, r=1,2,3$ is implicit, to include all the final lepton states. For $2 m_{S_{3}}>m_{\ell_{b}}+m_{\ell_{d}}+m_{h}$, the $\epsilon=L R$ 
operator also yields $S_{3} S_{3}^{*} \rightarrow \ell_{b}^{-} \ell_{d}^{+} h$, but its impact can be neglected in our $m_{S_{3}}$ range of interest.

Since the $\Delta$ 's in eq. (3.9) contain many free parameters, to proceed we need to make more specific choices regarding $C_{b d k l}^{L R, L, R}$. For simplicity, we adopt

$$
C_{b d k l}^{L R}=\frac{\sqrt{2} \kappa_{L R} m_{\ell_{d}}}{v} \delta_{b l} \delta_{d k}, \quad C_{b d k l}^{L}=2 \kappa_{L} \delta_{b l} \delta_{d k}, \quad C_{b d k l}^{R}=\kappa_{R} \delta_{b d} \delta_{k l},
$$

with $\kappa_{L R, L, R}$ being real constants. From eq. (4.7) and the unitarity of $\mathcal{U}$, we then have

$$
\mathcal{C}_{b d}^{L R}=\frac{\sqrt{2} \kappa_{L R} m_{\ell_{d}}}{v} \mathcal{U}_{b 3} \mathcal{U}_{d 3}^{*}, \quad \mathcal{C}_{b d}^{L}=2 \kappa_{L} \mathcal{U}_{b 3} \mathcal{U}_{d 3}^{*}, \quad \mathcal{C}_{b d}^{R}=\kappa_{R} \delta_{b d} .
$$

We also need to specify the $S_{k}$ masses. Among the different ways to realize A in eq. (2.10), we concentrate on the least complicated possibility that $O$ is a real orthogonal matrix, in addition to the right-handed neutrinos being degenerate with $M_{\nu}=\mathcal{M} \mathbb{1}$, in which case

$$
\mathrm{A}=\frac{2 \mathcal{M}}{v^{2}} U_{\mathrm{PMNS}} \hat{m}_{\nu} U_{\mathrm{PMNS}}^{\dagger} .
$$

With $\mathrm{A}=\mathcal{U} \operatorname{diag}\left(\hat{\mathrm{A}}_{1}, \hat{\mathrm{A}}_{2}, \hat{\mathrm{A}}_{3}\right) \mathcal{U}^{\dagger}$, this implies that

$$
\mathcal{U}=U_{\mathrm{PMNS}}, \quad \hat{\mathrm{A}}_{k}=\frac{2 \mathcal{M} m_{k}}{v^{2}} .
$$

The $S_{k}$ mass formula in eq. (3.7) then becomes

$$
m_{S_{k}}^{2}=\mu_{s 0}^{2}+\lambda_{s 0} v^{2}+\frac{2\left(\mu_{s 1}^{2}+\lambda_{s 1} v^{2}\right) \mathcal{M} m_{k}}{v^{2}}+\frac{4\left(\mu_{s 2}^{2}+\lambda_{s 2} v^{2}\right) \mathcal{M}^{2} m_{k}^{2}}{v^{4}},
$$

indicating that the pattern of $S_{k}$ masses is connected to the mass hierarchy of the light neutrinos. For definiteness, we pick

$$
\mu_{s 0}^{2}+\lambda_{s 0} v^{2}=\mu_{s 1}^{2}+\lambda_{s 1} v^{2}=\mu_{s 2}^{2}+\lambda_{s 2} v^{2}
$$

Thus a normal hierarchy of neutrino masses, $m_{1}<m_{2} \ll m_{3}$, would cause $S_{1,2}$ to be close in mass and lighter than $S_{3}$, implying that at least both $S_{1,2}$ determine the DM density. As stated earlier, here we examine the simpler scenario with the inverted hierarchy of neutrino masses, $m_{3} \ll m_{1}<m_{2}$, so that only $S_{3}$ is the DM and the heavier $S_{1,2}$ have negligible effects on the relic abundance.

For numerical computations below, we need to know the elements of $\mathcal{U}$ as well as the light neutrino eigenmasses. We employ the central values of the parameter ranges

$$
\begin{array}{rlrl}
\sin ^{2} \theta_{12} & =0.308 \pm 0.017, & \sin ^{2} \theta_{23}=0.455_{-0.031}^{+0.139}, \\
\sin ^{2} \theta_{13} & =0.0240_{-0.0022}^{+0.0019}, & \delta / \pi=1.31_{-0.33}^{+0.29}, \\
\delta m^{2} & =m_{2}^{2}-m_{1}^{2}=\left(7.54_{-0.22}^{+0.26}\right) \times 10^{-5} \mathrm{eV}^{2}, \\
\Delta m^{2} & =\frac{1}{2}\left(m_{1}^{2}+m_{2}^{2}\right)-m_{3}^{2}=\left(2.38_{-0.06}^{+0.06}\right) \times 10^{-3} \mathrm{eV}^{2}
\end{array}
$$




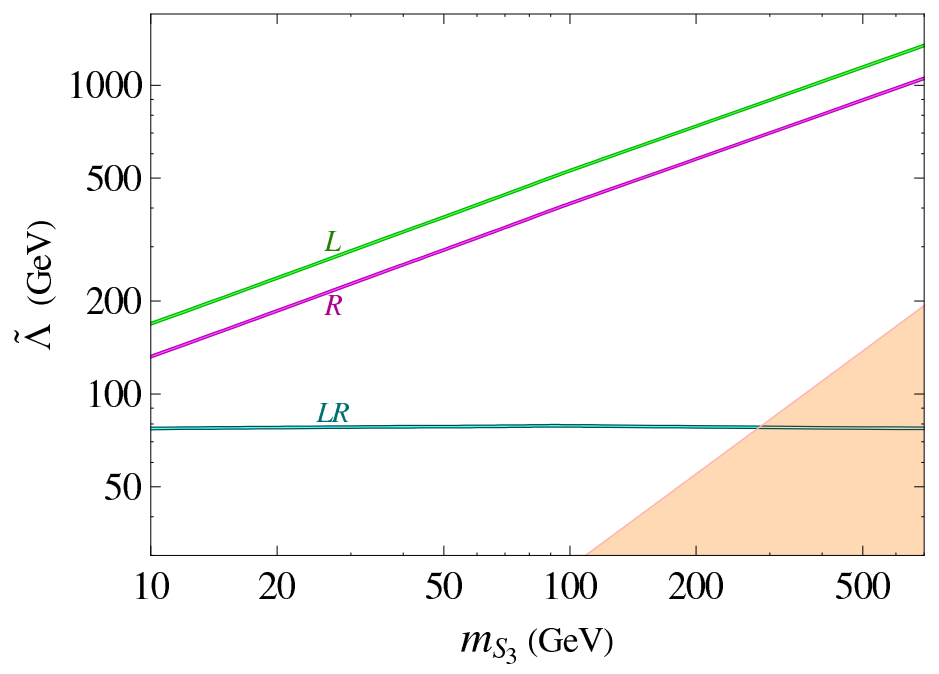

Figure 2. Values of $\tilde{\Lambda}=\Lambda /\left|\kappa_{\epsilon}\right|^{1 / 2}$ for $\epsilon=L, R, L R$ which fulfill the relic density constraint, as discussed in the text. In this and the following figures, the (orange) shaded region depicts the parameter space where the effective field theory approach breaks down.

from a recent fit to the global data on neutrino oscillation [60] in the case of inverted hierarchy of neutrino masses. Since empirical information on the absolute scale of $m_{1,2,3}$ is still far from precise [1], we set $m_{3}=0$. Requiring the largest eigenvalue of $\mathrm{A}$ in eq. (4.11) to be unity, we then get $\mathcal{M}=6.15 \times 10^{14} \mathrm{GeV}$. Applying these mass numbers and eq. (4.14) in eq. (4.13) results in $m_{S_{1}} \simeq 1.7 m_{S_{3}}$ and $m_{S_{1}, S_{2}}$ differing by $\sim 0.8 \%$.

We can now extract the values of $\tilde{\Lambda} \equiv \Lambda /\left|\kappa_{\epsilon}\right|^{1 / 2}$ that fulfill the relic density requirement using eq. (4.8) with the couplings given in eq. (4.10). Assuming that only one of $\kappa_{L R, L, R}$ is nonzero at a time and that the $\lambda_{3}$ contributions evaluated earlier are absent, we present the results in figure 2. The curve for $\epsilon=L R$ arises from $\hat{a}$ in eq. (4.8), with the contribution from $\hat{b}$ having been neglected, whereas the $\epsilon=L$ or $R$ curve comes from $\hat{b}$ alone. ${ }^{5}$ If the $\lambda_{3}$ contributions are also present and nonnegligible, and if they do not cancel the effectivecoupling contributions in the $\ell_{k}^{-} \ell_{k}^{+}$channels, there will be less room for each of the two sources, which will push the $\tilde{\Lambda}$ curves upward. On the lower right portion of the plot, we have also drawn an orange area, which satisfies $2 \pi \tilde{\Lambda}<m_{S_{2}}$ for the parameter choices in eq. (4.14) and the preceding paragraph. This region corresponds to the parameter space where the effective field theory description is no longer valid [61].

There is another restraint from DM data that should be mentioned. For $m_{S_{3}}<20 \mathrm{GeV}$, the predicted annihilation rate for the $\tau^{+} \tau^{-}$final-state is in some tension with upper limits inferred from searches for DM signals in diffuse gamma-ray data from the Fermi Large Area Telescope observations of dwarf spheroidal satellite galaxies of the Milky Way [62-64].

More significantly, complementary constraints on the effective lepton- $S$ couplings are available from experimental studies at LEP II on the monophoton production process $e^{+} e^{-} \rightarrow \gamma \notin$ with missing energy $\mathbb{E}^{\mathrm{E}}$ in the final state. These measurements were carried

\footnotetext{
${ }^{5}$ The roughly flat behavior of the $L R$ (blue) curve reflects the $m_{S_{3}}$ independence of $\hat{a}$ in eq. (4.8) for negligible lepton masses and is similar to its counterpart in the quark-flavored-DM scenario [14].
} 
out to examine the neutrino counting reaction $e^{+} e^{-} \rightarrow \gamma \nu \bar{\nu}$ in the SM and also to look for new particles that are long-lived or stable [65-76]. Thus the acquired data may be useful for restricting the process $e^{+} e^{-} \rightarrow \gamma S_{k} S_{l}^{*}$ if $S_{k, l}$ are long-lived or, for $k, l \neq 3$, if they decay (sequentially) into $S_{3}$ plus light neutrinos. This transition arises from two diagrams each containing an $e^{+} e^{-} \rightarrow S_{k} S_{l}^{*}$ vertex with the photon being radiated off the $e^{-}$or $e^{+}$ line. We have written down its amplitude and sketched the calculation of the cross section, $\sigma_{e \bar{e} \rightarrow \gamma S_{k} \bar{S}_{l}}$, in appendix A. Summing it over the final flavors then yields $\sigma_{e \bar{e} \rightarrow \gamma S \bar{S}^{\prime} \rightarrow \gamma \not^{E}}$ if $S_{k, l}$ are stable or long-lived. If they decay, we can express instead

$$
\sigma_{e \bar{e} \rightarrow \gamma S \bar{S}^{\prime} \rightarrow \gamma E^{\prime}}=\sum_{k, l=1}^{3} \sigma_{e \bar{e} \rightarrow \gamma S_{k} \bar{S}_{l}} \mathcal{B}_{k 3} \mathcal{B}_{l 3}
$$

with the branching ratios

$$
\mathcal{B}_{13}=\mathcal{B}\left(S_{1} \rightarrow \nu \nu^{\prime} S_{3}\right), \quad \mathcal{B}_{23}=\mathcal{B}\left(S_{2} \rightarrow \nu \nu^{\prime} S_{3}\right)+\mathcal{B}\left(S_{2} \rightarrow \nu \nu^{\prime} S_{1}\right) \mathcal{B}_{13}, \quad \mathcal{B}_{33}=1
$$

where the sum includes only kinematically allowed channels and $\mathcal{B}\left(S_{l} \rightarrow \nu \nu^{\prime} S_{k}\right)=$ $\Gamma_{S_{l} \rightarrow \nu \nu^{\prime} S_{k}} / \Gamma_{S_{l}}$ from the rates derived in appendix B.

The LEP II experiments on $e^{+} e^{-} \rightarrow \gamma \notin$ had c.m. energies within the range 130$207 \mathrm{GeV}$, and the observed cross-sections vary also with cuts on the photon energy $E_{\gamma}$ and angle $\theta_{\gamma}$ relative to the beam direction. From a collection of these data [65-76] tabulated in ref. [77], one can see that the majority of the measured and SM values of the cross section agree with each other at the one-sigma level. Consequently, to bound the eeS $S^{\prime}$ couplings, we may require $\sigma_{e \bar{e} \rightarrow \gamma S \bar{S}^{\prime} \rightarrow \gamma \mathcal{E}^{\prime}}$ not to exceed the corresponding one-sigma empirical errors (after combining the statistical and systematic errors in quadrature).

Applying this condition and assuming as before that only one of the $C$ couplings in eq. (3.9) is nonzero at a time, for the coupling choices in eq. (4.9) we find that $\kappa_{L R} / \Lambda^{2}$ does not get any meaningful limitations from the LEP II measurements, which is not unexpected because the resulting eeS $S^{\prime}$ interaction is suppressed by the electron mass, as eq. (A.4) indicates. On the other hand, they do translate into moderate restraints on $\kappa_{L(R)} / \Lambda^{2}$. More precisely, from the data, we infer the dotted curves shown in figure 3 which represent lower limits on $\tilde{\Lambda}$ and therefore reduce the parameter space consistent with the observed relic abundance (the solid thin bands), so that now $m_{S_{3}} \lesssim 24$ (43) GeV is excluded for $\epsilon=L(R)$. It is clear from this simple exercise that future $e^{+} e^{-}$machines with greater energies and luminosities, such as the International Linear Collider [78], can be expected to probe more stringently this new-physics scenario, if they detect no signals beyond the SM.

Another important implication of the dimension- 6 effective $\ell \ell^{\prime} S S^{\prime}$ interactions is that they can give rise to the flavor-changing decay $\ell_{a}^{-} \rightarrow \ell_{b}^{-} \ell_{c}^{-} \ell_{d}^{+}$via one-loop diagrams involving internal $S_{k, l}$ if at least one of the couplings is flavor violating. Such decays have been searched for over the years, but with null results so far, leading to increasingly severe bounds on their branching ratios [1]. Consequently, their data may give rise to substantial restrictions on the couplings.

We again assume that only one of the couplings in eq. (3.9) is contributing at a time. Since $C^{R}$ conserves flavor, only $C^{L R}$ and $C^{L}$ as specified in eq. (4.9) are pertinent. Thus 


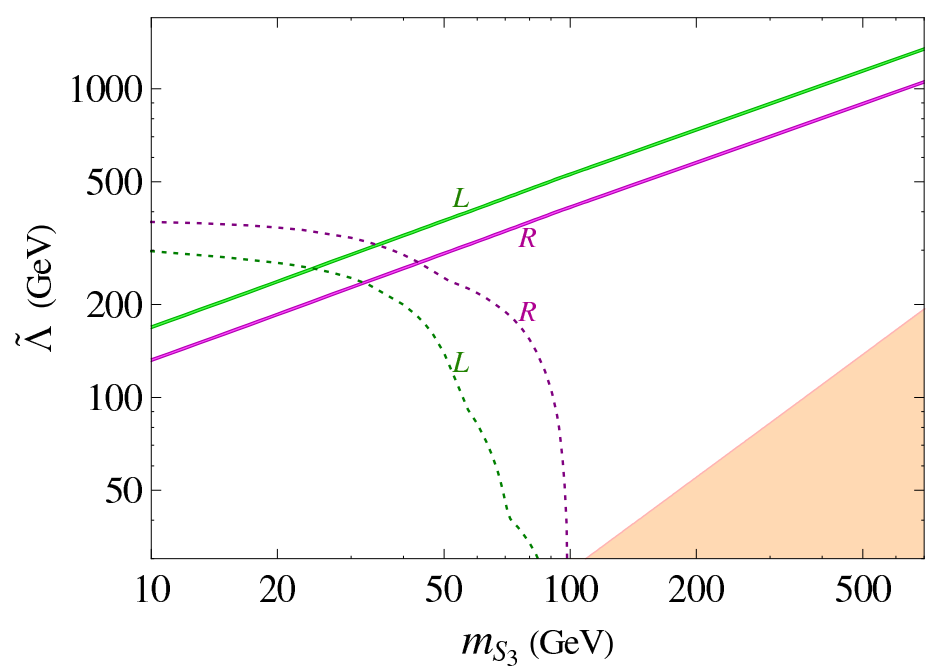

Figure 3. Values of $\tilde{\Lambda}=\Lambda /\left|\kappa_{\epsilon}\right|^{1 / 2}$ for $\epsilon=L$ and $R$ which are compatible with the observed relic abundance (solid thin bands), compared to lower limits on $\tilde{\Lambda}$ inferred from measurements of $e^{+} e^{-} \rightarrow \gamma \notin$ at LEP II (dotted curves).

we can express the amplitudes for $\ell_{a}^{-} \rightarrow \ell_{b}^{-} \ell_{c}^{-} \ell_{d}^{+}$in each case as

$$
\begin{aligned}
\mathcal{M}_{\ell_{a} \rightarrow \ell_{b} \ell_{c} \bar{\ell}_{d}}^{L R}= & \frac{\mathcal{I}\left(m_{S_{k}}, m_{S_{l}}\right) v^{2}}{16 \pi^{2} \Lambda^{4}} \mathcal{U}_{p k}^{*} \mathcal{U}_{s k} \mathcal{U}_{q l} \mathcal{U}_{r l}^{*} \\
& \times\left[\bar{u}_{c}\left(C_{c a p q}^{L R} P_{R}+C_{a c q p}^{L R *} P_{L}\right) u_{a} \bar{u}_{b}\left(C_{b d r s}^{L R} P_{R}+C_{d b s r}^{L R *} P_{L}\right) v_{d}-(b \leftrightarrow c)\right], \\
\mathcal{M}_{\ell_{a} \rightarrow \ell_{b} \ell_{c} \bar{\ell}_{d}}^{L}= & \frac{\mathcal{J}\left(m_{S_{k}}, m_{S_{l}}\right)}{8 \pi^{2} \Lambda^{4}} \mathcal{U}_{p k}^{*} \mathcal{U}_{r k} \mathcal{U}_{q l} \mathcal{U}_{s l}^{*}\left(C_{c a p q}^{L} C_{b d s r}^{L}+C_{b a p q}^{L} C_{c d s r}^{L}\right) \bar{u}_{c} \gamma^{\rho} P_{L} u_{a} \bar{u}_{b} \gamma_{\rho} P_{L} v_{d},
\end{aligned}
$$

where $k, l, p, q, r, s=1,2,3$ are summed over and and $\mathcal{I}$ and $\mathcal{J}$ are loop functions. With the choices of $C^{L R, L}$ in eq. (4.9), we arrive at

$$
\mathcal{I}(m, n)=\frac{m^{2} \ln (n / m)}{m^{2}-n^{2}}, \quad \mathcal{J}(m, n)=m^{2}\left(\ln \frac{\Lambda}{n}+\frac{1}{4}\right)+\frac{m^{4} \ln (n / m)}{m^{2}-n^{2}},
$$

where we have dropped terms that vanished after $k$ is summed over in eqs. (4.18) and (4.19) due to $a \neq b, c, d$ and the unitarity of $\mathcal{U}$. We have also taken the cutoff in the loop integration to be the same as the scale $\Lambda$ and neglected the momenta of the external particles.

Upon comparing the resulting branching ratio of $\ell_{a}^{-} \rightarrow \ell_{b}^{-} \ell_{c}^{-} \ell_{d}^{+}$to its measured bound, one can then derive a limit on $\Lambda /\left|\kappa_{L R}\right|^{1 / 2}$, assuming that only $C^{L R}$ is nonzero. The relevant modes are $\mu^{-} \rightarrow e^{-} e^{-} e^{+}$and $\tau^{-} \rightarrow$ $e^{-} e^{-} e^{+}, \mu^{-} \mu^{-} \mu^{+}, e^{-} e^{-} \mu^{+}, \mu^{-} \mu^{-} e^{+}, \mu^{-} e^{-} e^{+}, e^{-} \mu^{-} \mu^{+}$, for which only experimental bounds on the branching ratios are available. Although the strictest among them is $\mathcal{B}\left(\mu^{-} \rightarrow e^{-} e^{-} e^{+}\right)_{\exp }<1.0 \times 10^{-12}$, we find that $\mathcal{B}\left(\tau^{-} \rightarrow \mu^{-} \mu^{-} \mu^{+}\right) \exp <2.1 \times 10^{-8}[1]$ yields the strongest constraint, namely

$$
\frac{\Lambda}{\left|\kappa_{L R}\right|^{1 / 2}}>11 \mathrm{GeV}
$$




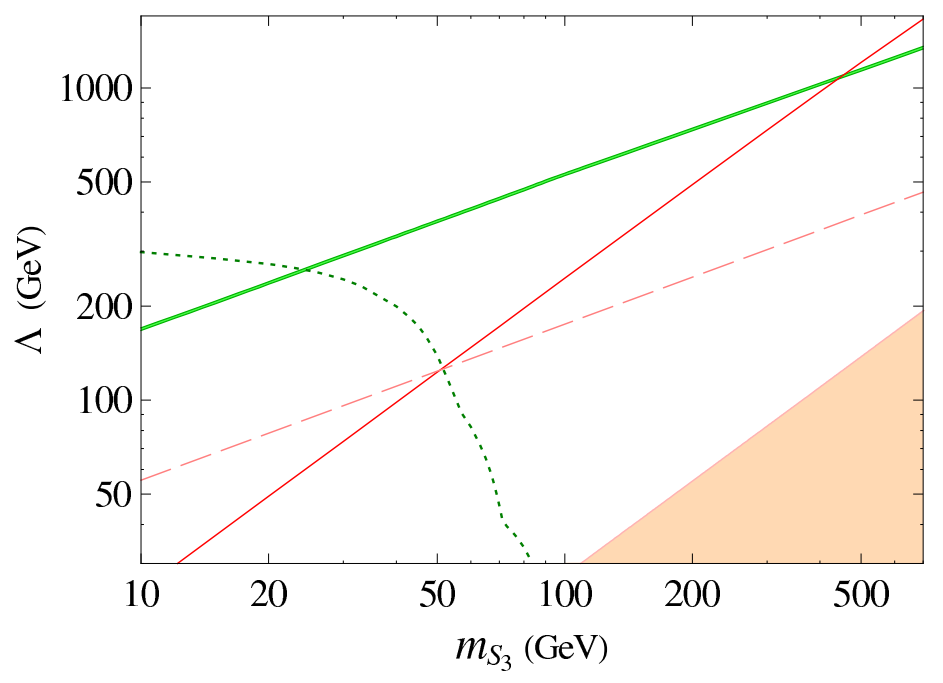

Figure 4. Values of $\Lambda$ for $C^{L}$ in eq. (4.9) with $\kappa_{L}=1$ which fulfill the relic density constraint (green thin band), compared to the lower limits on $\Lambda$ from the LEP II data on $e^{+} e^{-} \rightarrow \gamma \notin$ (green dotted curve) and from searches for $\mu^{-} \rightarrow e^{-} e^{-} e^{+}$(red solid curve) and $\tau^{-} \rightarrow \mu^{-} \mu^{-} e^{+}$(red dashed curve).

which is consistent with the $L R$ curve in figure 2. This is mainly due to the enhancement from the lepton mass factor in the rate of $\tau^{-} \rightarrow \mu^{-} \mu^{-} \mu^{+}$, as can be seen from the expressions for $\ell^{\prime-} \rightarrow \ell^{-} \ell^{-} \ell^{+}$rates collected in appendix $\mathrm{C}$.

If instead only $C^{L}$ in eq. (4.9) is present, $\mathcal{B}\left(\mu^{-} \rightarrow e^{-} e^{-} e^{+}\right)$exp turns out to impose the most stringent constraint among these decays in the $m_{S_{3}} \gtrsim 50 \mathrm{GeV}$ region, whereas for lower masses $\mathcal{B}\left(\tau^{-} \rightarrow \mu^{-} \mu^{-} e^{+}\right)_{\exp }<1.7 \times 10^{-8} \quad[1]$ is the most restrictive. The formulas for their rates are also listed in appendix C. In figure 4 we depict the resulting lower-limits on $\Lambda$. In this case, we set $\kappa_{L}=1$ due to the $\ln \Lambda$ dependence of the $\mu^{-} \rightarrow e^{-} e^{-} e^{+}$rate. The plot reveals that above $m_{S_{3}} \sim 500 \mathrm{GeV}$ the $\Lambda$ values consistent with the observed relic density are in conflict with the bound from the $\mu^{-} \rightarrow e^{-} e^{-} e^{+}$data. This significantly shrinks the allowed parameter space already decreased by the restraint from the LEP II measurements.

\subsection{Flavor-violating Higgs decay}

The recently discovered Higgs boson can potentially offer a window into physics beyond the SM. The presence of new particles can bring about modifications to the standard decay modes of the Higgs and/or cause it to undergo exotic decays [79]. As data from the LHC continues to accumulate with increasing precision, they may uncover clues of new physics in the Higgs couplings.

The CMS collaboration [80] has recently reported the detection of a slight excess of $h \rightarrow$ $\mu^{ \pm} \tau^{\mp}$ events with a significance of $2.5 \sigma$. If interpreted as a signal, the result corresponds to a branching fraction of $\mathcal{B}(h \rightarrow \mu \tau)=\mathcal{B}\left(h \rightarrow \mu^{-} \tau^{+}\right)+\mathcal{B}\left(h \rightarrow \mu^{+} \tau^{-}\right)=\left(0.89_{-0.37}^{+0.40}\right) \%$, but as a statistical fluctuation it translates into a limit of $\mathcal{B}(h \rightarrow \mu \tau)<1.57 \%$ at 95\% CL [80]. It is too early to draw a definite conclusion from this finding, but it would constitute clear 
evidence of physics beyond the SM if substantiated by future measurements. Assuming that the tentative signal hint is true, we investigate whether the $S_{k}$ interactions could effect such an exotic Higgs decay within the allowed parameter space. ${ }^{6}$

One can write the amplitude for $h \rightarrow \ell_{b}^{-} \ell_{d}^{+}$as

$$
\mathcal{M}_{h \rightarrow \ell_{b} \bar{\ell}_{d}}=\frac{y_{b d}^{\mathrm{SM}}+y_{b d}^{\mathrm{new}}}{v} \bar{u}_{b}\left(m_{\ell_{b}} P_{L}+m_{\ell_{d}} P_{R}\right) v_{d}
$$

corresponding to the rate

$$
\Gamma_{h \rightarrow \ell_{b} \bar{\ell}_{d}}=\frac{m_{h}\left|y_{b d}^{\mathrm{SM}}+y_{b d}^{\mathrm{new}}\right|^{2}}{16 \pi v^{2}}\left(m_{\ell_{b}}^{2}+m_{\ell_{d}}^{2}\right)
$$

where $y_{b d}^{\mathrm{SM}}=\delta_{b d}$ in the SM at tree level and $y_{b d}^{\text {new }}$ stands for the new contribution. The main contribution to $y_{b d}^{\text {new }}$ originates from a one-loop diagram involving internal $S_{k}$, one $h S_{k} S_{k}$ vertex from the renormalizable Lagrangian in eq. (3.6), and one $S_{k} S_{k} \ell \ell^{\prime}$ vertex from a dimension-six operator in eq. (3.8). It turns out that for the latter coupling only $O^{L R}$ matters, as the loop contributions of $O^{L, R}$ vanish. Thus, focusing on the case in which $m_{h}<2 m_{S_{k}}$ and $C^{L R}$ is given by eq. (4.9), we obtain

$$
y_{b d}^{\text {new }}=\frac{\kappa_{L R} \lambda_{k} \mathcal{U}_{d k}^{*} \mathcal{U}_{b k} v^{2}}{16 \pi^{2} \Lambda^{2}}\left(\ln \frac{\Lambda^{2}}{m_{S_{k}}^{2}}+1-2 \sqrt{\frac{4 m_{S_{k}}^{2}}{m_{h}^{2}}-1} \sin ^{-1} \frac{m_{h}}{2 m_{S_{k}}}\right),
$$

where summation over $k=1,2,3$ is implicit and we have again taken the cutoff in the loop integration to be the same as $\Lambda$. The $S_{k} S_{k} \ell \ell^{\prime}$ coupling alone can generate oneloop contributions to the off-diagonal elements of the charged-lepton mass matrix, but we estimate their impact on its eigenvalues to be small. Therefore, $y_{b d}^{\text {new }}$ in eq. (4.24) is largely unaffected as the leading contribution of $S_{k}$ to $h \rightarrow \ell_{b}^{-} \ell_{d}^{+}$.

Since $h \rightarrow \tau^{+} \tau^{-}, \mu^{+} \mu^{-}$also receive the $S_{k}$ contributions in eq. (4.24), we need to take into account the relevant data. The ATLAS and CMS collaborations have reported the observations of $h \rightarrow \tau^{+} \tau^{-}$and measured its signal strength to be $\sigma / \sigma_{\mathrm{SM}}=1.42_{-0.38}^{+0.44}$ and $0.91 \pm 0.27$, respectively $[52,85]$. In contrast, the only experimental information on $h \rightarrow \mu^{+} \mu^{-}$are the bounds $\mathcal{B}(h \rightarrow \mu \bar{\mu})<1.5 \times 10^{-3}$ and $1.6 \times 10^{-3}$ from ATLAS and CMS, respectively $[86,87]$. In view of these data, we demand the $S_{k}$ contributions to respect

$$
0.7<\frac{\Gamma_{h \rightarrow \tau \bar{\tau}}}{\Gamma_{h \rightarrow \tau \bar{\tau}}^{\mathrm{SM}}}<1.8, \quad \frac{\Gamma_{h \rightarrow \mu \bar{\mu}}}{\Gamma_{h \rightarrow \mu \bar{\mu}}^{\mathrm{SM}}}<6.7,
$$

where $\Gamma_{h \rightarrow \tau \bar{\tau}}^{\mathrm{SM}}=257 \mathrm{keV}$ and $\Gamma_{h \rightarrow \mu \bar{\mu}}^{\mathrm{SM}}=894 \mathrm{eV}[53]$ for $m_{h}=125.1 \mathrm{GeV}$.

Due to the $\ln \Lambda$ dependence of $y_{b d}^{\text {new }}$, we also set $\kappa_{L R}=1$. It follows that, for illustration, we can select $\left(m_{S_{3}}, \Lambda\right)=(70,79)$ and $(200,78) \mathrm{GeV}$ from the $L R$ (blue) curve in figure 2, implying that we have assumed $\lambda_{3}$ to be negligible. Choosing also $\lambda_{1}=\lambda_{2}$ for simplification, we find that for $\left(m_{S_{3}}, \Lambda\right)=(70,79) \mathrm{GeV}$ the $S_{k}$ contributions lead to $0.0026>\left|y_{\mu \tau, \tau \mu}^{\text {new }}\right| m_{\tau} / v>0.0021$, or $0.79 \%>\mathcal{B}(h \rightarrow \mu \tau)>0.52 \%$, compatible with the range of the CMS finding on the potential signal [80], if $-7.2<\lambda_{1}<-5.8$. For

\footnotetext{
${ }^{6}$ The CMS excess has also been addressed in the contexts of other new-physics scenarios [81-84].
} 
$\left(m_{S_{3}}, \Lambda\right)=(200,78) \mathrm{GeV}$, we obtain the same $\mathcal{B}(h \rightarrow \mu \tau)$ range if $-2.9<\lambda_{1}<-2.4$. All these numbers correspond to $1.6<\Gamma_{h \rightarrow \tau \bar{\tau}} / \Gamma_{h \rightarrow \tau \bar{\tau}}^{\mathrm{SM}}<1.8$ and $1.8<\Gamma_{h \rightarrow \mu \bar{\mu}} / \Gamma_{h \rightarrow \mu \bar{\mu}}^{\mathrm{SM}}<2.0$, which conform to the conditions in eq. (4.25) and are therefore testable soon with forthcoming data from the LHC. Moreover, we determine that $\Gamma_{h \rightarrow e \tau}=0.053 \Gamma_{h \rightarrow \mu \tau}$. Although the preferred values of $\left|\lambda_{1,2}\right|$ seem to be sizable, they are still below the perturbativity limit of $4 \pi$ mentioned earlier. It is worth noting that the $\left|y_{\mu \tau, \tau \mu}^{\text {new }}\right| m_{\tau} / v$ numbers above are below the upper limit of 0.016 inferred from the measured bound on the $\tau \rightarrow \mu \gamma$ decay [88-90].

We have seen from the limited exercises performed in this paper that the MFV framework offers a systematic way to explore potential relations between DM, neutrinos, and the Higgs boson through a variety of processes which can be checked experimentally. More sophisticated choices of the coefficients $C^{L, R, L R}$ than those in eq. (4.9) would then allow the examination of a greater number of leptonic observables.

\section{Conclusions}

We have considered DM which is a singlet under the SM gauge group and a member of a scalar triplet under the lepton flavor group. The triplet is odd under an extra $Z_{2}$ symmetry which renders the DM candidate stable. We apply the MFV principle to all the lepton-flavored particles in the theory which includes three right-handed neutrinos taking part in the seesaw mechanism for neutrino mass generation. The new scalars couple to SM particles via Higgs-portal renormalizable interactions and dimension-six operators involving leptons. The MFV framework allows us to make interesting phenomenological connections between the DM, Higgs, and lepton sectors. We examine restrictions on the new scalars from the Higgs boson data, observed relic density, DM direct searches, LEP II measurements on $e^{+} e^{-}$scattering into a photon plus missing energy, and experimental bounds on flavor-violating lepton decays. We obtain viable parameter space that can be probed further by future experiments. Our simple choices of the new scalars' effective couplings illustrate how various data can constrain them in complementary ways. We also explore whether the scalar interactions can account for the tentative hint of the Higgs flavor-violating decay $h \rightarrow \mu \tau$ recently detected in the CMS experiment. Their contributions, occurring at the one-loop level, can give rise to a decay rate compatible with the CMS finding and at the same time fulfill requirements from other Higgs data. If it is not confirmed by upcoming measurements, the acquired data will place stronger limitations on the considered scenario of lepton-flavored DM with MFV. Last but not least, it is clear from our analysis that next-generation $e^{+} e^{-}$machines with high energies and luminosities, such as the International Linear Collider, have considerable potential for testing different aspects of this kind of new physics in greater detail.

\section{Acknowledgments}

We would like to thank Xiao-Gang He for helpful comments. We also thank Brian Batell for remarks concerning the stability of lepton-flavored dark matter. This work was supported 
in part by the MOE Academic Excellence Program (Grant No. 102R891505) and the NCTS.

\section{A Cross section of $e^{+} e^{-} \rightarrow \gamma S_{k} S_{l}^{*}$}

For the scattering $e^{-}(p) e^{+}(\bar{p}) \rightarrow \gamma(\mathrm{K}) S_{k}(q) S_{l}^{*}(\bar{q})$, we define the Lorentz-invariant kinematical variables

$$
\begin{aligned}
& s=(p+\bar{p})^{2}, \quad s^{\prime}=(q+\bar{q})^{2}, \quad t=(\bar{p}-\bar{q})^{2}, \quad t^{\prime}=(p-q)^{2}, \\
& u=(\bar{p}-q)^{2}, \quad u^{\prime}=(p-\bar{q})^{2}, \quad w=2 \mathrm{~K} \cdot p, \quad \bar{w}=2 \mathrm{~K} \cdot \bar{p} .
\end{aligned}
$$

We derive its amplitude $\mathcal{M}_{e \bar{e} \rightarrow \gamma S_{k} \bar{S}_{l}}$ from two diagrams each with an $e^{-} e^{+} \rightarrow S_{k} S_{l}^{*}$ vertex and the photon radiated from the $e^{-}$or $e^{+}$leg. Thus, in the limit of massless $e^{ \pm}$,

$$
\begin{aligned}
\mathcal{M}_{e \bar{e} \rightarrow \gamma S_{k} \bar{S}_{l}}= & \frac{\sqrt{4 \alpha \pi}}{\Lambda^{2}} \bar{v}_{\bar{e}}\left[(\not-\not l)\left(\mathrm{C}_{k l}^{L} P_{L}+\mathrm{C}_{k l}^{R} P_{R}\right)-\frac{v}{\sqrt{2}}\left(\mathrm{C}_{k l}^{L R} P_{R}+\overline{\mathrm{C}}_{k l}^{L R} P_{L}\right)\right] \frac{\not p-\not k}{w} \ddagger^{*} u_{e} \\
& -\frac{\sqrt{4 \alpha \pi}}{\Lambda^{2}} \bar{v}_{\bar{e} \ddagger^{*}} \frac{\not p-\not k}{\bar{w}}\left[(\phi-\not l)\left(\mathrm{C}_{k l}^{L} P_{L}+\mathrm{C}_{k l}^{R} P_{R}\right)-\frac{v}{\sqrt{2}}\left(\mathrm{C}_{k l}^{L R} P_{R}+\overline{\mathrm{C}}_{k l}^{L R} P_{L}\right)\right] u_{e},
\end{aligned}
$$

where $\alpha=1 / 128$ is the fine-structure constant,

$$
\mathrm{C}_{k l}^{\epsilon}=\sum_{n, o} \mathcal{U}_{k n}^{\dagger} \mathcal{U}_{o l} C_{11 n o}^{\epsilon}, \quad \epsilon=L, R, L R, \quad \overline{\mathrm{C}}_{k l}^{L R}=\left(\mathrm{C}_{l k}^{L R}\right)^{*} .
$$

Hence for the choices in eq. (4.9)

$$
\mathrm{C}_{k l}^{L}=2 \kappa_{L} \mathcal{U}_{1 k}^{*} \mathcal{U}_{1 l}, \quad \mathrm{C}_{k l}^{R}=\kappa_{R} \delta_{k l}, \quad \mathrm{C}_{k l}^{L R}=\frac{\sqrt{2} \kappa_{L R} m_{e}}{v} \mathcal{U}_{1 k}^{*} \mathcal{U}_{1 l} .
$$

It is easy to check that $\mathcal{M}_{e \bar{e} \rightarrow \gamma S_{k} \bar{S}_{l}}$ respects electromagnetic gauge invariance. Averaging (summing) its absolute square over the initial (final) spins, one then obtains

$$
\begin{aligned}
\overline{\left|\mathcal{M}_{e \bar{e} \rightarrow \gamma S_{k} \bar{S}_{l}}\right|^{2}=} & \frac{2 \alpha \pi\left(\left|\mathrm{C}_{k l}^{L}\right|^{2}+\left|\mathrm{C}_{k l}^{R}\right|^{2}\right)}{\Lambda^{4} w \bar{w}}\left\{2\left(m_{S_{k}}^{2}-m_{S_{l}}^{2}\right)\left[m_{S_{k}}^{2} s-m_{S_{l}}^{2} s+(t-u) w-\left(t^{\prime}-u^{\prime}\right) \bar{w}\right]\right. \\
& \left.+\left(w^{2}+\bar{w}^{2}+2 s s^{\prime}\right)\left(s^{\prime}-2 m_{S_{k}}^{2}-2 m_{S_{l}}^{2}\right)-s^{\prime}(t-u)^{2}-s^{\prime}\left(t^{\prime}-u^{\prime}\right)^{2}\right\} \\
& +\frac{\alpha \pi v^{2}}{\Lambda^{4} w \bar{w}}\left(\left|\mathrm{C}_{k l}^{L R}\right|^{2}+\left|\mathrm{C}_{l k}^{L R}\right|^{2}\right)\left(s^{2}+s^{\prime 2}\right) .
\end{aligned}
$$

This leads to the cross section

$$
\sigma_{e \bar{e} \rightarrow \gamma S_{k} \bar{S}_{l}}=\int \frac{E_{\gamma} d E_{\gamma} d\left(\cos \theta_{\gamma}\right) d \bar{\Omega}_{S}}{2(4 \pi)^{4} s} \sqrt{1-\frac{2 m_{S_{k}}^{2}+2 m_{S_{l}}^{2}}{s-2 E_{\gamma} \sqrt{s}}+\left(\frac{m_{S_{k}}^{2}-m_{S_{l}}^{2}}{s-2 E_{\gamma} \sqrt{s}}\right)^{2}} \frac{\left.\mathcal{M}_{e \bar{e} \rightarrow \gamma S_{k} \bar{S}_{l}}\right|^{2}}{},
$$

where $E_{\gamma}$ and $\theta_{\gamma}$ are the photon energy and angle with respect to the $e^{-}$or $e^{+}$beam direction in the c.m. frame of the $e^{+} e^{-}$pair, $\bar{\Omega}_{S}$ denotes the solid angle of either $S_{k}$ or $S_{l}^{*}$ in the c.m. frame of the $S_{k} S_{l}^{*}$ pair. The photon energy range is

$$
E_{\gamma}^{\min } \leq E_{\gamma} \leq E_{\gamma}^{\max }=\frac{s-\left(m_{S_{k}}+m_{S_{l}}\right)^{2}}{2 \sqrt{s}}
$$


where $E_{\gamma}^{\min }$ is an experimental cut. In the numerical evaluation of the integral, the $\theta_{\gamma}$ range is also subject to cuts.

It is worth mentioning that one could alternatively estimate $\sigma_{e \bar{e} \rightarrow \gamma S_{k} \bar{S}_{l}}$ in the so-called radiator approximation $[91,92]$. It is given by

$$
\begin{gathered}
\sigma_{e \bar{e} \rightarrow \gamma S_{k} \bar{S}_{l}}=\int d c_{\gamma} d x_{\gamma} \mathcal{H}\left(c_{\gamma}, x_{\gamma} ; s\right) \hat{\sigma}(\hat{s}), \\
c_{\gamma}=\cos \theta_{\gamma}, \quad x_{\gamma}=\frac{2 E_{\gamma}}{\sqrt{s}}, \quad \mathcal{H}\left(c_{\gamma}, x_{\gamma} ; s\right)=\frac{\alpha}{\pi} \frac{\left(2-x_{\gamma}\right)^{2}+c_{\gamma}^{2} x_{\gamma}^{2}}{2\left(1-c_{\gamma}^{2}\right) x_{\gamma}}, \quad \hat{s}=s-s x_{\gamma},
\end{gathered}
$$

where $\hat{\sigma}(\hat{s})$ stands for the cross section of the simpler reaction $e^{+} e^{-} \rightarrow S_{k} S_{l}^{*}$,

$$
\hat{\sigma}(\hat{s})=\frac{\mathcal{K}^{\frac{3}{2}}\left(\hat{s}, m_{S_{k}}^{2}, m_{S_{l}}^{2}\right)}{96 \Lambda^{4} \pi \hat{s}^{2}}\left(\left|\mathrm{C}_{k l}^{L}\right|^{2}+\left|\mathrm{C}_{k l}^{R}\right|^{2}\right)+\frac{\mathcal{K}^{\frac{1}{2}}\left(\hat{s}, m_{S_{k}}^{2}, m_{S_{l}}^{2}\right) v^{2}}{128 \Lambda^{4} \pi \hat{s}}\left(\left|\mathrm{C}_{k l}^{L R}\right|^{2}+\left|\mathrm{C}_{l k}^{L R}\right|^{2}\right) .
$$

With this method, the $\mathrm{C}_{k l}^{L, R}$ contributions to $\sigma_{e \bar{e} \rightarrow \gamma S_{k} \bar{S}_{l}}$ turn out to be exactly the same as their counterparts in eq. (A.6), whereas the $\mathrm{C}_{k l}^{L R}$ terms would yield numbers lower by no more than several percent.

\section{B Decays of $S$ particles}

The decay of $S_{l}$ into $S_{k}$ plus charged leptons if kinematically permitted may arise from the operators $O^{L, R, L R}$ in eq. (3.8), depending on the specifics of the couplings. For $O^{L}$, the final leptons can also be neutrinos instead. The amplitudes for $S_{l}(q) \rightarrow S_{k}(p) \ell_{b}^{-} \ell_{d}^{+}$and $S_{l}(q) \rightarrow S_{k}(p) \nu_{b} \nu_{d}$ are then

$$
\begin{aligned}
\mathcal{M}_{S_{l} \rightarrow \ell_{b} \bar{\ell}_{d} S_{k}} & =\frac{-1}{\Lambda^{2}} \bar{u}_{b}\left[\frac{v}{\sqrt{2}}\left(\mathrm{c}_{b d k l}^{L R} P_{R}+\overline{\mathrm{c}}_{b d k l}^{L R} P_{L}\right)+(p+q)_{\rho} \gamma^{\rho}\left(\mathrm{c}_{b d k l}^{L} P_{L}+\mathrm{c}_{b d k l}^{R} P_{R}\right)\right] v_{d}, \\
\mathcal{M}_{S_{l} \rightarrow \nu_{b} \nu_{d} S_{k}} & =\frac{-1}{\Lambda^{2}}(p+q)_{\rho} \bar{u}_{b} \gamma^{\rho}\left(\mathrm{c}_{b d k l}^{L} P_{L}-\mathrm{c}_{d b k l}^{L} P_{R}\right) v_{d}
\end{aligned}
$$

where

$$
c_{b d k l}^{\epsilon}=\sum_{n, o} \mathcal{U}_{k n}^{\dagger} \mathcal{U}_{o l} C_{b d n o}^{\epsilon}, \quad \overline{\mathrm{c}}_{b d k l}^{L R}=\left(\mathrm{c}_{d b l k}^{L R}\right)^{*}
$$

Thus for the choices in eq. (4.9)

$$
c_{b d k l}^{L}=2 \kappa_{L} \mathcal{U}_{d k}^{*} \mathcal{U}_{b l}, \quad c_{b d k l}^{R}=\kappa_{R} \delta_{b d} \delta_{k l}, \quad c_{b d k l}^{L R}=\frac{\sqrt{2} \kappa_{L R} m_{\ell_{d}}}{v} \mathcal{U}_{d k}^{*} \mathcal{U}_{b l},
$$


and so with the above $\mathrm{c}_{b d k l}^{R}$ alone $S_{1,2,3}$ are all stable. From eqs. (B.1) and (B.2), the decay rates for negligible lepton masses are

$$
\begin{aligned}
\Gamma_{S_{l} \rightarrow \ell_{b} \bar{\ell}_{d} S_{k}}= & \frac{\left(\left|\mathrm{c}_{b d k l}^{L R}\right|^{2}+\left|\mathrm{c}_{d b l k}^{L R}\right|^{2}\right) v^{2}}{3072 \Lambda^{4} \pi^{3} m_{S_{l}}^{3}}\left[\left(m_{S_{l}}^{2}-m_{S_{k}}^{2}\right)\left(m_{S_{l}}^{4}+10 m_{S_{k}}^{2} m_{S_{l}}^{2}+m_{S_{k}}^{4}\right)\right. \\
& \left.-12 m_{S_{k}}^{2} m_{S_{l}}^{2}\left(m_{S_{k}}^{2}+m_{S_{l}}^{2}\right) \ln \frac{m_{S_{l}}}{m_{S_{k}}}\right] \\
& +\frac{\left|\mathrm{c}_{b d k l}^{L}\right|^{2}+\left|\mathrm{c}_{b d k l}^{R}\right|^{2}}{1536 \Lambda^{4} \pi^{3} m_{S_{l}}^{3}}\left[\left(m_{S_{l}}^{4}-m_{S_{k}}^{4}\right)\left(m_{S_{l}}^{4}-8 m_{S_{k}}^{2} m_{S_{l}}^{2}+m_{S_{k}}^{4}\right)+24 m_{S_{k}}^{4} m_{S_{l}}^{4} \ln \frac{m_{S_{l}}}{m_{S_{k}}}\right], \\
\Gamma_{S_{l} \rightarrow \nu \nu^{\prime} S_{k}}= & \frac{1}{2} \sum_{b, d} \Gamma_{S_{l} \rightarrow \nu_{b} \nu_{d} S_{k}} \\
= & \frac{\sum_{b, d}\left|c_{b d k l}^{L}\right|^{2}}{1536 \Lambda^{4} \pi^{3} m_{S_{l}}^{3}}\left[\left(m_{S_{l}}^{4}-m_{S_{k}}^{4}\right)\left(m_{S_{l}}^{4}-8 m_{S_{k}}^{2} m_{S_{l}}^{2}+m_{S_{k}}^{4}\right)+24 m_{S_{k}}^{4} m_{S_{l}}^{4} \ln \frac{m_{S_{l}}}{m_{S_{k}}}\right],
\end{aligned}
$$

where the factor of $\frac{1}{2}$ in $\Gamma_{S_{l} \rightarrow \nu \nu^{\prime} S_{k}}$ accounts for the identical Majorana neutrinos in the final states of channels with $b=d$ and prevents double counting of contributions with $b \neq d$. In the numerical evaluation of $\Gamma_{S_{l} \rightarrow \ell_{b} \bar{\ell}_{d} S_{k}}$, we do not neglect the lepton masses. For our $m_{S_{k}}$ choices, these three-body modes dominate the total widths of $S_{1,2}$, and so we can approximate them to be $\Gamma_{S_{1}}=\Gamma_{S_{1} \rightarrow \nu \nu^{\prime} S_{3}}+\Gamma_{S_{1} \rightarrow \bar{\ell}^{\prime} S_{3}}$ and $\Gamma_{S_{2}}=\Gamma_{S_{2} \rightarrow \nu \nu^{\prime} S_{1}}+\Gamma_{S_{2} \rightarrow \nu \nu^{\prime} S_{3}}+$ $\Gamma_{S_{2} \rightarrow \ell \bar{\ell}^{\prime} S_{1}}+\Gamma_{S_{2} \rightarrow \ell \bar{\ell} S_{3}}$, where $\Gamma_{S_{l} \rightarrow \ell \bar{\ell}^{\prime} S_{k}}=\sum_{b, d} \Gamma_{S_{l} \rightarrow \ell \ell_{b} \bar{\ell}_{d} S_{k}}$, excluding kinematically forbidden channels.

\section{Rates of $\ell^{\prime-} \rightarrow \ell^{-} \ell^{+} \ell^{-}$}

The rate of the flavor-violating decay $\ell^{\prime-} \rightarrow \ell^{-} \ell^{-} \ell^{+}$induced by the $\kappa_{L R}$ contribution alone from eq. (4.9) can be expressed as

$$
\Gamma_{\ell^{\prime} \rightarrow \ell \ell \bar{\ell}}^{L R}=\frac{\left|\kappa_{L R}\right|^{4}\left|k_{\ell^{\prime} \rightarrow \ell \ell \bar{\ell}}^{L R}\right|^{2} m_{\ell^{\prime}}^{7} m_{\ell}^{2}}{4096 \pi^{3}}
$$

where the lepton mass $m_{\ell}$ in the final state has been neglected in the phase-space integration. For $\mu^{-} \rightarrow e^{-} e^{-} e^{+}$and $\tau^{-} \rightarrow \mu^{-} \mu^{-} \mu^{+}$, we derive, respectively,

$$
\begin{aligned}
& k_{\mu \rightarrow e e \bar{e}}^{L R}=\left[\left(\frac{m_{S_{1}}^{2}+m_{S_{3}}^{2}}{m_{S_{1}}^{2}-m_{S_{3}}^{2}} \ln \frac{m_{S_{1}}}{m_{S_{3}}}-1\right)\left(1-2 s_{13}^{2}\right)-\ln \frac{m_{S_{1}}}{m_{S_{3}}}\right] \frac{c_{13} s_{13} s_{23}}{16 \pi^{2} \Lambda^{4}}, \\
& k_{\tau \rightarrow \mu \mu \bar{\mu}}^{L R}=\left[\left(\frac{m_{S_{1}}^{2}+m_{S_{3}}^{2}}{m_{S_{1}}^{2}-m_{S_{3}}^{2}} \ln \frac{m_{S_{1}}}{m_{S_{3}}}-1\right)\left(1-2 c_{13}^{2} s_{23}^{2}\right)-\ln \frac{m_{S_{1}}}{m_{S_{3}}}\right] \frac{c_{13}^{2} c_{23} s_{23}}{16 \pi^{2} \Lambda^{4}},
\end{aligned}
$$

upon making the approximation $m_{S_{1}}=m_{S_{2}}$.

Similarly, the rate of $\ell^{\prime-} \rightarrow \ell_{1}^{-} \ell_{1}^{-} \ell_{2}^{+}$due to $\kappa_{L}$ alone from eq. (4.9) is

$$
\Gamma_{\ell^{\prime} \rightarrow \ell_{1}^{-} \ell_{1}^{-} \ell_{2}^{+}}^{L}=\frac{\left|\kappa_{L}\right|^{4}\left|k_{\ell^{\prime} \rightarrow \ell_{1} \ell_{1} \bar{\ell}_{2}}^{L}\right|^{2} m_{\ell^{\prime}}^{5}}{3072 \pi^{3}}
$$


For $\mu^{-} \rightarrow e^{-} e^{-} e^{+}$and $\tau^{-} \rightarrow \mu^{-} \mu^{-} e^{+}$, we get, respectively,

$$
\begin{aligned}
k_{\mu \rightarrow e e \bar{e}}^{L}= & {\left[\left(\frac{m_{S_{1}}^{2} m_{S_{3}}^{2}}{m_{S_{1}}^{2}-m_{S_{3}}^{2}} \ln \frac{m_{S_{1}}}{m_{S_{3}}}-\frac{m_{S_{1}}^{2}+m_{S_{3}}^{2}}{4}\right)\left(1-2 s_{13}^{2}\right)\right.} \\
& \left.+m_{S_{1}}^{2} \ln \frac{\Lambda}{m_{S_{1}}}-m_{S_{3}}^{2} \ln \frac{\Lambda}{m_{S_{3}}}\right] \frac{c_{13} s_{13} s_{23}}{\pi^{2} \Lambda^{4}}, \\
k_{\tau \rightarrow \mu \mu \bar{e}}^{L}= & \left(\frac{m_{S_{1}}^{2} m_{S_{3}}^{2}}{m_{S_{1}}^{2}-m_{S_{3}}^{2}} \ln \frac{m_{S_{1}}}{m_{S_{3}}}-\frac{m_{S_{1}}^{2}+m_{S_{3}}^{2}}{4}\right) \frac{2 c_{13}^{3} s_{13} c_{23} s_{23}^{2}}{\pi^{2} \Lambda^{4}} .
\end{aligned}
$$

Open Access. This article is distributed under the terms of the Creative Commons Attribution License (CC-BY 4.0), which permits any use, distribution and reproduction in any medium, provided the original author(s) and source are credited.

\section{References}

[1] Particle Data Group ollaboration, K.A. Olive et al., Review of Particle Physics (RPP), Chin. Phys. C 38 (2014) 090001 [inSPIRE].

[2] G. Bertone, D. Hooper and J. Silk, Particle dark matter: Evidence, candidates and constraints, Phys. Rept. 405 (2005) 279 [hep-ph/0404175] [INSPIRE].

[3] D. Hooper and E.A. Baltz, Strategies for Determining the Nature of Dark Matter, Ann. Rev. Nucl. Part. Sci. 58 (2008) 293 [arXiv:0802.0702] [INSPIRE].

[4] J.L. Feng, Dark Matter Candidates from Particle Physics and Methods of Detection, Ann. Rev. Astron. Astrophys. 48 (2010) 495 [arXiv: 1003.0904] [INSPIRE].

[5] R.S. Chivukula and H. Georgi, Composite Technicolor Standard Model, Phys. Lett. B 188 (1987) 99 [INSPIRE].

[6] L.J. Hall and L. Randall, Weak scale effective supersymmetry, Phys. Rev. Lett. 65 (1990) 2939 [inSPIRE].

[7] A.J. Buras, P. Gambino, M. Gorbahn, S. Jager and L. Silvestrini, Universal unitarity triangle and physics beyond the standard model, Phys. Lett. B 500 (2001) 161 [hep-ph/0007085] [INSPIRE].

[8] A.J. Buras, Minimal flavor violation, Acta Phys. Polon. B 34 (2003) 5615 [hep-ph/0310208] [INSPIRE].

[9] S. Davidson and F. Palorini, Various definitions of Minimal Flavour Violation for Leptons, Phys. Lett. B 642 (2006) 72 [hep-ph/0607329] [INSPIRE].

[10] A.L. Kagan, G. Perez, T. Volansky and J. Zupan, General Minimal Flavor Violation, Phys. Rev. D 80 (2009) 076002 [arXiv:0903.1794] [INSPIRE].

[11] A.J. Buras and J. Girrbach, Towards the Identification of New Physics through Quark Flavour Violating Processes, Rept. Prog. Phys. 77 (2014) 086201 [arXiv:1306.3775] [INSPIRE].

[12] G. D'Ambrosio, G.F. Giudice, G. Isidori and A. Strumia, Minimal flavor violation: An Effective field theory approach, Nucl. Phys. B 645 (2002) 155 [hep-ph/0207036] [INSPIRE]. 
[13] V. Cirigliano, B. Grinstein, G. Isidori and M.B. Wise, Minimal flavor violation in the lepton sector, Nucl. Phys. B 728 (2005) 121 [hep-ph/0507001] [INSPIRE].

[14] B. Batell, J. Pradler and M. Spannowsky, Dark matter from minimal flavor violation, JHEP 08 (2011) 038 [arXiv:1105.1781] [INSPIRE].

[15] B. Batell, T. Lin and L.-T. Wang, Flavored dark matter and R-parity violation, JHEP 01 (2014) 075 [arXiv:1309.4462] [INSPIRE].

[16] P. Minkowski, $\mu \rightarrow$ er at a Rate of One Out of $10^{9}$ Muon Decays?, Phys. Lett. B 67 (1977) 421 [INSPIRE].

[17] T. Yanagida, in Proceedings of the Workshop on the Unified Theory and the Baryon Number in the Universe, O. Sawada and A. Sugamoto eds., KEK, Tsukuba Japan (1979), pg. 95.

[18] T. Yanagida, Horizontal Symmetry and Masses of Neutrinos, Prog. Theor. Phys. 64 (1980) 1103 [InSPIRE].

[19] M. Gell-Mann, P. Ramond and R. Slansky, Complex Spinors And Unified Theories, in Supergravity, P. van Nieuwenhuizen and D. Freedman eds., North-Holland, Amsterdam The Netherlands (1979), pg. 315.

[20] P. Ramond, The Family Group in Grand Unified Theories, hep-ph/9809459 [INSPIRE].

[21] S.L. Glashow, in Proceedings of the 1979 Cargese Summer Institute on Quarks and Leptons, M. Levy et al. eds., Plenum Press, New York U.S.A. (1980), pg. 687.

[22] R.N. Mohapatra and G. Senjanović, Neutrino Mass and Spontaneous Parity Violation, Phys. Rev. Lett. 44 (1980) 912 [InSPIRE].

[23] J. Schechter and J.W.F. Valle, Neutrino Masses in $\mathrm{SU}(2) \times \mathrm{U}(1)$ Theories, Phys. Rev. D 22 (1980) 2227 [inSPIRE].

[24] J. Schechter and J.W.F. Valle, Neutrino Decay and Spontaneous Violation of Lepton Number, Phys. Rev. D 25 (1982) 774 [INSPIRE].

[25] L. Lopez-Honorez and L. Merlo, Dark matter within the minimal flavour violation ansatz, Phys. Lett. B 722 (2013) 135 [arXiv:1303.1087] [INSPIRE].

[26] B. Pontecorvo, Neutrino Experiments and the Problem of Conservation of Leptonic Charge, Sov. Phys. JETP 26 (1968) 984 [INSPIRE].

[27] Z. Maki, M. Nakagawa and S. Sakata, Remarks on the unified model of elementary particles, Prog. Theor. Phys. 28 (1962) 870 [inSPIRE].

[28] J.A. Casas and A. Ibarra, Oscillating neutrinos and $\mu \rightarrow e, \gamma$, Nucl. Phys. B 618 (2001) 171 [hep-ph/0103065] [INSPIRE].

[29] G. Colangelo, E. Nikolidakis and C. Smith, Supersymmetric models with minimal flavour violation and their running, Eur. Phys. J. C 59 (2009) 75 [arXiv:0807.0801] [InSPIRE].

[30] L. Mercolli and C. Smith, EDM constraints on flavored CP-violating phases, Nucl. Phys. B 817 (2009) 1 [arXiv:0902.1949] [INSPIRE].

[31] X.-G. He, C.-J. Lee, S.-F. Li and J. Tandean, Large electron electric dipole moment in minimal flavor violation framework with Majorana neutrinos, Phys. Rev. D 89 (2014) 091901 [arXiv: 1401.2615] [InSPIRE].

[32] X.-G. He, C.-J. Lee, S.-F. Li and J. Tandean, Fermion EDMs with Minimal Flavor Violation, JHEP 08 (2014) 019 [arXiv: 1404.4436] [INSPIRE]. 
[33] P. Agrawal, S. Blanchet, Z. Chacko and C. Kilic, Flavored Dark Matter and Its Implications for Direct Detection and Colliders, Phys. Rev. D 86 (2012) 055002 [arXiv:1109.3516] [INSPIRE].

[34] J. Kile, Flavored Dark Matter: A Review, Mod. Phys. Lett. A 28 (2013) 1330031 [arXiv: 1308.0584] [INSPIRE].

[35] A. Hamze, C. Kilic, J. Koeller, C. Trendafilova and J.-H. Yu, Lepton-Flavored Asymmetric Dark Matter and Interference in Direct Detection, Phys. Rev. D 91 (2015) 035009 [arXiv: 1410.3030] [INSPIRE].

[36] M. Hirsch, S. Morisi, E. Peinado and J.W.F. Valle, Discrete dark matter, Phys. Rev. D 82 (2010) 116003 [arXiv: 1007.0871] [INSPIRE].

[37] D. Meloni, S. Morisi and E. Peinado, Neutrino phenomenology and stable dark matter with A4, Phys. Lett. B 697 (2011) 339 [arXiv:1011.1371] [INSPIRE].

[38] M.S. Boucenna, M. Hirsch, S. Morisi, E. Peinado, M. Taoso et al., Phenomenology of Dark Matter from $A_{4}$ Flavor Symmetry, JHEP 05 (2011) 037 [arXiv:1101.2874] [INSPIRE].

[39] M.S. Boucenna, S. Morisi, E. Peinado, Y. Shimizu and J.W.F. Valle, Predictive discrete dark matter model and neutrino oscillations, Phys. Rev. D 86 (2012) 073008 [arXiv:1204.4733] [INSPIRE].

[40] S. Kanemura, T. Kasai and Y. Okada, Mass bounds of the lightest CP even Higgs boson in the two Higgs doublet model, Phys. Lett. B 471 (1999) 182 [hep-ph/9903289] [INSPIRE].

[41] G. Cynolter, E. Lendvai and G. Pocsik, Note on unitarity constraints in a model for a singlet scalar dark matter candidate, Acta Phys. Polon. B 36 (2005) 827 [hep-ph/0410102] [INSPIRE].

[42] J.F. Kamenik and C. Smith, FCNC portals to the dark sector, JHEP 03 (2012) 090 [arXiv:1111.6402] [INSPIRE].

[43] E.W. Kolb and M. Turner, Frontiers in Physics. Vol. 69: The Early Universe, Westview Press, Boulder U.S.A. (1990).

[44] V. Silveira and A. Zee, Scalar phantoms, Phys. Lett. B 161 (1985) 136 [inSPIRE].

[45] J. McDonald, Gauge singlet scalars as cold dark matter, Phys. Rev. D 50 (1994) 3637 [hep-ph/0702143] [INSPIRE].

[46] C.P. Burgess, M. Pospelov and T. ter Veldhuis, The minimal model of nonbaryonic dark matter: a singlet scalar, Nucl. Phys. B 619 (2001) 709 [hep-ph/0011335] [INSPIRE].

[47] V. Barger, P. Langacker, M. McCaskey, M. Ramsey-Musolf and G. Shaughnessy, Complex Singlet Extension of the Standard Model, Phys. Rev. D 79 (2009) 015018 [arXiv:0811.0393] [INSPIRE].

[48] J.M. Cline, K. Kainulainen, P. Scott and C. Weniger, Update on scalar singlet dark matter, Phys. Rev. D 88 (2013) 055025 [arXiv: 1306.4710] [INSPIRE].

[49] X.-G. He, S.-Y. Ho, J. Tandean and H.-C. Tsai, Scalar Dark Matter and Standard Model with Four Generations, Phys. Rev. D 82 (2010) 035016 [arXiv: 1004.3464] [INSPIRE].

[50] X.-G. He, B. Ren and J. Tandean, Hints of Standard Model Higgs Boson at the LHC and Light Dark Matter Searches, Phys. Rev. D 85 (2012) 093019 [arXiv:1112.6364] [INSPIRE]. 
[51] ATLAS collaboration, Measurement of the Higgs boson mass from the $H \rightarrow \gamma \gamma$ and $H \rightarrow Z Z^{*} \rightarrow 4 \ell$ channels with the ATLAS detector using $25 \mathrm{fb}^{-1}$ of pp collision data, Phys. Rev. D 90 (2014) 052004 [arXiv: 1406. 3827] [INSPIRE].

[52] CMS collaboration, Precise determination of the mass of the Higgs boson and studies of the compatibility of its couplings with the standard model, CMS-PAS-HIG-14-009 (2014).

[53] https://twiki.cern.ch/twiki/bin/view/LHCPhysics/CERNYellowReportPageBR3.

[54] LUX collaboration, D.S. Akerib et al., First results from the LUX dark matter experiment at the Sanford Underground Research Facility, Phys. Rev. Lett. 112 (2014) 091303 [arXiv: 1310.8214] [INSPIRE].

[55] A. Falkowski, F. Riva and A. Urbano, Higgs at last, JHEP 11 (2013) 111 [arXiv:1303.1812] [INSPIRE].

[56] P.P. Giardino, K. Kannike, I. Masina, M. Raidal and A. Strumia, The universal Higgs fit, JHEP 05 (2014) 046 [arXiv:1303.3570] [INSPIRE].

[57] J. Ellis and T. You, Updated Global Analysis of Higgs Couplings, JHEP 06 (2013) 103 [arXiv: 1303.3879] [INSPIRE].

[58] G. Bélanger, B. Dumont, U. Ellwanger, J.F. Gunion and S. Kraml, Global fit to Higgs signal strengths and couplings and implications for extended Higgs sectors, Phys. Rev. D 88 (2013) 075008 [arXiv:1306.2941] [InSPIRE].

[59] K. Cheung, J.S. Lee and P.-Y. Tseng, Higgs precision analysis updates 2014, Phys. Rev. D 90 (2014) 095009 [arXiv:1407.8236] [INSPIRE].

[60] F. Capozzi et al., Status of three-neutrino oscillation parameters, circa 2013, Phys. Rev. D 89 (2014) 093018 [arXiv: 1312.2878] [INSPIRE].

[61] J. Goodman et al., Constraints on Dark Matter from Colliders, Phys. Rev. D 82 (2010) 116010 [arXiv: 1008.1783] [InSPIRE].

[62] Fermi-LAT collaboration, M. Ackermann et al., Constraining Dark Matter Models from a Combined Analysis of Milky Way Satellites with the Fermi Large Area Telescope, Phys. Rev. Lett. 107 (2011) 241302 [arXiv:1108.3546] [INSPIRE].

[63] A. Geringer-Sameth and S.M. Koushiappas, Exclusion of canonical WIMPs by the joint analysis of Milky Way dwarfs with Fermi, Phys. Rev. Lett. 107 (2011) 241303 [arXiv: 1108.2914] [INSPIRE].

[64] A. Geringer-Sameth, S.M. Koushiappas and M.G. Walker, A Comprehensive Search for Dark Matter Annihilation in Dwarf Galaxies, arXiv:1410.2242 [INSPIRE].

[65] ALEPH collaboration, D. Buskulic et al., A study of single and multi-photon production in $e^{+} e^{-}$collisions at center-of-mass energies of 130-GeV and 136-GeV, Phys. Lett. B 384 (1996) 333 [inSPIRE].

[66] ALEPH collaboration, R. Barate et al., Searches for supersymmetry in the photon(s) plus missing energy channels at $\sqrt{s}=161 \mathrm{GeV}$ and $172 \mathrm{GeV}$, Phys. Lett. B 420 (1998) 127 [hep-ex/9710009] [INSPIRE].

[67] ALEPH collaboration, R. Barate et al., Single photon and multiphoton production in $e^{+} e^{-}$ collisions at a center-of-mass energy of $183 \mathrm{GeV}$, Phys. Lett. B 429 (1998) 201 [INSPIRE].

[68] ALEPH collaboration, A. Heister et al., Single photon and multiphoton production in $e^{+} e^{-}$ collisions at $\sqrt{s}$ up to $209 \mathrm{GeV}$, Eur. Phys. J. C 28 (2003) 1 [INSPIRE]. 
[69] DELPHI collaboration, P. Abreu et al., Photon events with missing energy at $\sqrt{s}=183$ GeV to 189 GeV, Eur. Phys. J. C 17 (2000) 53 [hep-ex/0103044] [InSPIRE].

[70] DELPHI collaboration, J. Abdallah et al., Photon events with missing energy in $e^{+} e^{-}$ collisions at $\sqrt{s}=130 \mathrm{GeV}$ to $209 \mathrm{GeV}$, Eur. Phys. J. C 38 (2005) 395 [hep-ex/0406019] [INSPIRE].

[71] L3 collaboration, M. Acciarri et al., Single and multiphoton events with missing energy in $e^{+} e^{-}$collisions at $161 \mathrm{GeV}<\sqrt{s}<172 \mathrm{GeV}$, Phys. Lett. B 415 (1997) 299 [INSPIRE].

[72] L3 collaboration, M. Acciarri et al., Single and multiphoton events with missing energy in $e^{+} e^{-}$collisions at $\sqrt{s}=183$ GeV, Phys. Lett. B 444 (1998) 503 [InSPIRE].

[73] L3 collaboration, M. Acciarri et al., Single and multiphoton events with missing energy in $e^{+} e^{-}$collisions at $\sqrt{S}-189-G e V$, Phys. Lett. B 470 (1999) 268 [hep-ex/9910009] [INSPIRE].

[74] OPAL collaboration, K. Ackerstaff et al., Search for anomalous production of photonic events with missing energy in $e^{+} e^{-}$collisions at $\sqrt{s}=130 \mathrm{GeV}$ to $172 \mathrm{GeV}$, Eur. Phys. J. C 2 (1998) 607 [hep-ex/9801024] [InSPIRE].

[75] OPAL collaboration, G. Abbiendi et al., Search for anomalous photonic events with missing energy in $e^{+} e^{-}$collisions at $\sqrt{s}=130 \mathrm{GeV}, 136 \mathrm{GeV}$ and $183 \mathrm{GeV}$, Eur. Phys. J. C 8 (1999) 23 [hep-ex/9810021] [INSPIRE].

[76] OPAL collaboration, G. Abbiendi et al., Photonic events with missing energy in $e^{+} e^{-}$ collisions at $\sqrt{s}=189$ GeV, Eur. Phys. J. C 18 (2000) 253 [hep-ex/0005002] [INSPIRE].

[77] C.-W. Chiang, G. Faisel, Y.-F. Lin and J. Tandean, Constraining Nonstandard Neutrino-Electron Interactions due to a New Light Spin-1 Boson, JHEP 10 (2013) 150 [arXiv:1204.6296] [INSPIRE].

[78] T. Behnke et al., The International Linear Collider Technical Design Report - Volume 1: Executive Summary, arXiv:1306.6327 [INSPIRE].

[79] D. Curtin et al., Exotic decays of the 125 GeV Higgs boson, Phys. Rev. D 90 (2014) 075004 [arXiv:1312.4992] [INSPIRE].

[80] CMS collaboration, Search for Lepton Flavour Violating Decays of the Higgs Boson, CMS-PAS-HIG-14-005 (2014).

[81] A. Dery, A. Efrati, Y. Nir, Y. Soreq and V. Susič, Model building for flavor changing Higgs couplings, Phys. Rev. D 90 (2014) 115022 [arXiv:1408.1371] [INSPIRE].

[82] M.D. Campos, A.E.C. Hernández, H. Päs and E. Schumacher, Higgs $\rightarrow \mu \tau$ as an indication for $S_{4}$ flavor symmetry, arXiv:1408.1652 [INSPIRE].

[83] A. Celis, V. Cirigliano and E. Passemar, Disentangling new physics contributions in lepton flavour violating tau decays, arXiv: 1409.4439 [INSPIRE].

[84] D. Aristizabal Sierra and A. Vicente, Explaining the CMS Higgs flavor violating decay excess, Phys. Rev. D 90 (2014) 115004 [arXiv: 1409.7690] [INSPIRE].

[85] ATLAS collaboration, Evidence for Higgs boson Yukawa couplings in the $H \rightarrow \tau \tau$ decay mode with the ATLAS detector, ATLAS-CONF-2014-061 (2014).

[86] ATLAS collaboration, Search for the Standard Model Higgs boson decay to $\mu^{+} \mu^{-}$with the ATLAS detector, Phys. Lett. B 738 (2014) 68 [arXiv:1406.7663] [INSPIRE].

[87] CMS collaboration, Search for a standard model-like Higgs boson in the $\mu^{+} \mu^{-}$and $e^{+} e^{-}$ decay channels at the LHC, Phys. Lett. B 744 (2015) 184 [arXiv:1410.6679] [InSPIRE]. 
[88] A. Goudelis, O. Lebedev and J.-h. Park, Higgs-induced lepton flavor violation, Phys. Lett. B 707 (2012) 369 [arXiv:1111.1715] [INSPIRE].

[89] G. Blankenburg, J. Ellis and G. Isidori, Flavour-Changing Decays of a $125 \mathrm{GeV}$ Higgs-like Particle, Phys. Lett. B $\mathbf{7 1 2}$ (2012) 386 [arXiv:1202.5704] [INSPIRE].

[90] R. Harnik, J. Kopp and J. Zupan, Flavor Violating Higgs Decays, JHEP 03 (2013) 026 [arXiv: 1209.1397] [INSPIRE].

[91] O. Nicrosini and L. Trentadue, Structure Function Approach to the Neutrino Counting Problem, Nucl. Phys. B 318 (1989) 1 [INSPIRE].

[92] G. Montagna, O. Nicrosini, F. Piccinini and L. Trentadue, Invisible events with radiative photons at LEP, Nucl. Phys. B 452 (1995) 161 [hep-ph/9506258] [INSPIRE]. 